\title{
Dispersal Alters the Nature and Scope of Sexually Antagonistic Variation
}

\author{
Ewan O. Flintham, ${ }^{1}$ Vincent Savolainen, ${ }^{1, \star}$ and Charles Mullon ${ }^{2}$
}

1. Department of Life Sciences, Imperial College London, Silwood Park Campus, Ascot SL5 7PY, United Kingdom; 2. Department of Ecology and Evolution, University of Lausanne, 1015 Lausanne, Switzerland

Submitted May 14, 2020; Accepted December 1, 2020; Electronically published April 7, 2021

Online enhancements: appendixes, SLiM code.

\begin{abstract}
A S ST RACT: Intralocus sexual conflict, or sexual antagonism, occurs when alleles have opposing fitness effects in the two sexes. Previous theory suggests that sexual antagonism is a driver of genetic variation by generating balancing selection. However, most of these studies assume that populations are well mixed, neglecting the effects of spatial subdivision. Here, we use mathematical modeling to show that limited dispersal changes evolution at sexually antagonistic autosomal and X-linked loci as a result of inbreeding and sex-specific kin competition. We find that if the sexes disperse at different rates, kin competition within the philopatric sex biases intralocus conflict in favor of the more dispersive sex. Furthermore, kin competition diminishes the strength of balancing selection relative to genetic drift, reducing genetic variation in small subdivided populations. Meanwhile, by decreasing heterozygosity, inbreeding reduces the scope for sexually antagonistic polymorphism due to nonadditive allelic effects, and this occurs to a greater extent on the $\mathrm{X}$ chromosome than autosomes. Overall, our results indicate that spatial structure is a relevant factor in predicting where sexually antagonistic alleles might be observed. We suggest that sex-specific dispersal ecology and demography can contribute to interspecific and intragenomic variation in sexual antagonism.
\end{abstract}

Keywords: polymorphism, balancing selection, kin selection, sexspecific dispersal, genetic drift.

\section{Introduction}

As a result of the different reproductive roles of males and females, many traits are selected in different directions in the two sexes (Parker 1979). Responding to these divergent selection pressures, however, is not straightforward. Because the sexes share a large part of their genomes and traits are typically determined by the same genes, homologous traits in males and females tend to be genetically correlated (Poissant et al. 2010). Opposing selection pres-

\footnotetext{
* Corresponding author; email: v.savolainen@imperial.ac.uk. ORCIDs: Flintham, https://orcid.org/0000-0002-8037-5560; Savolainen, https://orcid.org/0000-0001-5350-9984.
}

Am. Nat. 2021. Vol. 197, pp. 543-559. (C) 2021 by The University of Chicago. 0003-0147/2021/19705-59969\$15.00. All rights reserved.

DOI: $10.1086 / 713739$ sures on the two sexes therefore lead to a genetic tug-ofwar, whereby some alleles are favored in one sex but disfavored in the other (Connallon and Clark 2014). This tug-of-war, also known as intralocus sexual conflict or sexual antagonism (Chippindale et al. 2001; Brommer et al. 2007; Foerster et al. 2007; Bonduriansky and Chenoweth 2009; Mainguy et al. 2009; Svensson et al. 2009; Lewis et al. 2011), can result in balancing selection and the long-term maintenance of polymorphism, within and even between species (e.g., Eyer et al. 2019; Ruzicka et al. 2019).

Mathematical population genetics models have helped elucidate the conditions that favor the emergence and maintenance of sexually antagonistic variation (e.g., Owen 1953; Kidwell et al. 1977; Rice 1984; Albert and Otto 2005; Gavrilets and Rice 2006; Fry 2010; Arnqvist 2011; Connallon and Clark 2012; Jordan and Charlesworth 2012; Mullon et al. 2012; Jaquiéry et al. 2013; Harts et al. 2014; Jordan and Connallon 2014; Tazzyman and Abbott 2015; Connallon et al. 2019; de Vries and Caswell 2019; Kasimatis et al. 2019; Hitchcock and Gardner 2020; Ruzicka and Connallon 2020). First, models have highlighted the role of dominance (i.e., nonadditive effects of alleles) in facilitating balancing selection at sexually antagonistic loci on both autosomes and sex chromosomes (Kidwell et al. 1977; Rice 1984; Fry 2010; Spencer and Priest 2016). In line with this prediction for autosomes, a number of empirical studies have found that loci underlying intralocus sexual conflict often exhibit sex differences in dominance that increase the fitness of both male and female heterozygotes (referred to as "dominance reversal"; Hager et al. 2008; Barson et al. 2015; Grieshop and Arnqvist 2018; Pearse et al. 2019). Second, stochastic models have emphasized the importance of genetic drift in countering the effects of sexually antagonistic selection in finite populations. In fact, because sexually antagonistic selection is relatively weak, its effects on polymorphism can be easily negated by genetic drift (Connallon and Clark 2012; Mullon et al. 2012).

A common assumption in the vast majority of models investigating sexually antagonistic variation is that 
populations are well mixed or that individuals compete at random (Owen 1953; Kidwell et al. 1977; Rice 1984; Albert and Otto 2005; Gavrilets and Rice 2006; Fry 2010; Connallon and Clark 2012; Jordan and Charlesworth 2012; Mullon et al. 2012; Jaquiéry et al. 2013; Jordan and Connallon 2014; de Vries and Caswell 2019; Ruzicka and Connallon 2020). In reality, most natural populations are spatially structured or subdivided, simply as a result of the physical constraints of movement that lead to limited dispersal (Clobert et al. 2001). Where dispersal is limited, individuals interacting with one another are more likely to share common alleles than individuals sampled at random in the population (Rousset 2004). Studies of sexual antagonism in a spatial context have so far ignored the effects of such genetic correlations (e.g., by assuming patches of infinite size [Connallon et al. 2019]; although for a general population genetics model of a dioecious subdivided population applied to imprinting, see Van Cleve et al. 2010). Genetic structure, however, has important evolutionary implications, as it leads to inbreeding and kin selection (Hamilton 1964; Frank 1998; Rousset 2004; Charlesworth and Charlesworth 2010). These effects may be particularly relevant for the fate of sexually antagonistic alleles, as inbreeding influences the abundance of heterozygotes and hence how dominance affects selection at sexually antagonistic loci (Arnqvist 2011; Jordan and Connallon 2014; Tazzyman and Abbott 2015; Kasimatis et al. 2019). In addition, limited dispersal influences the effective size of a population and thus the strength of genetic drift (Caballero 1994; Wang and Caballero 1999; Rousset 2004). But in spite of its potential importance, the consequences of population subdivision for sexually antagonistic variation remain poorly understood.

Here, we investigate the effects of limited dispersal on the segregation of sexually antagonistic alleles. To do this, we extend panmictic population genetics models of intralocus sexual conflict (e.g., Owen 1953; Kidwell et al. 1977; Fry 2010) to a subdivided population consisting of patches, or groups, interconnected by dispersal (e.g., Van Cleve et al. 2010). This model allows for sex-specific dispersal and demography (i.e., different dispersal rates for males and females and different numbers of breeding males and females within groups), as reported in many taxa (for reviews, see Trochet et al. 2016; Li and Kokko 2019). We examine how the interplay between selection, sex-specific ecology, and genetic drift influences the segregation of sexually antagonistic alleles at autosomal and sex-linked positions.

\section{Model}

Following Van Cleve et al. (2010), we consider a dioecious population that is subdivided among patches, each composed of $n_{\mathrm{m}}$ male and $n_{\mathrm{f}}$ female adults, with the following life cycle. First, within each patch, adults mate at random, producing juveniles of each sex in equal proportion. After reproduction, adults die. Second, each juvenile either remains in its natal patch or disperses to another randomly chosen patch (so dispersal is uniform among patches, as in the island model of Wright 1931). Juveniles disperse independently from one another, with a sex-specific probability, $m_{\mathrm{m}}$ and $m_{\mathrm{f}}$ in males and females, respectively. Third, within each patch and each sex, juveniles compete to fill $n_{\mathrm{m}}$ male and $n_{\mathrm{f}}$ female breeding positions in the local mating pool and become the adults of the next generation. With dispersal occurring prior to competition, our model considers hard selection (so that patches can vary in the number of adult offspring they contribute to the next generation; Roze and Rousset 2003; Débarre and Gandon 2011; see app. A, sec. A.2.6, for an analysis of our model under soft selection; apps. A, B are available online).

In line with previous studies of sexual antagonism (e.g., Owen 1953; Kidwell et al. 1977; Fry 2010), we consider a genetic locus, either on an autosome or on a sex chromosome (X in XY species or Z in ZW species), with two segregating alleles, $a$ and $A$. These alleles have opposing effects on male and female competitiveness (the third step of the life cycle): allele $a$ increases competitiveness when present in males but decreases competitiveness when present in females; conversely, allele $A$ improves female competitiveness but comes at a cost when in males (where the cost to male and female homozygotes carrying detrimental alleles is given by $c_{\mathrm{m}}$ and $c_{\mathrm{f}}$, respectively; fig. $1 A$ ). So the fittest males are homozygous $a a$, while the fittest females are $A A$. In $A a$ heterozygotes, the costs of the antagonistic alleles are mediated by sex-specific dominance coefficients, $h_{\mathrm{m}}$ in males and $h_{\mathrm{f}}$ in females, allowing for a variety of dominance effects, including dominance reversal (fig. $1 B-1 D$ ).

\section{Results \\ The Effects of Limited Dispersal on Sexually Antagonistic Selection}

We first explore the effect of limited dispersal on sexually antagonistic selection by considering mathematically the change in average frequency (weighted by reproductive value; e.g., Roze and Rousset 2003, eq. [35]; Van Cleve et al. 2010, eq. [2]) of the "male-detrimental" or "femalebeneficial" allele $A$. For tractability, our analysis initially assumes weak selection (i.e., $0<c_{\mathrm{m}}, c_{\mathrm{f}} \ll 1$ ), large female fecundity (large enough to ignore demographic stochasticity), and an effectively infinite number of patches (for derivation, see app. A).

Limited Dispersal Introduces Kin Selection and Inbreeding. We find that the change $\Delta p(p)$ in average frequency $p$ 


\section{A. Male and female phenotypes}

\section{B. Additive effects}

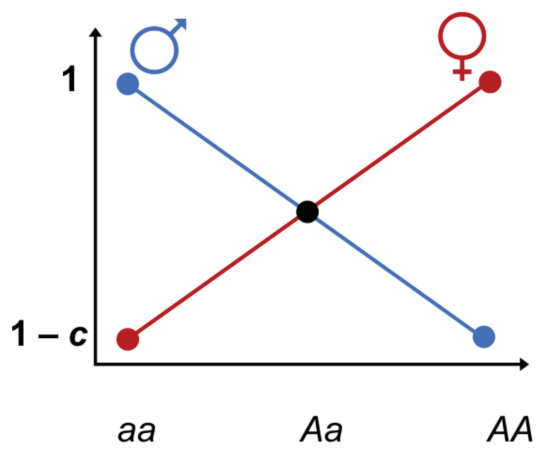

D. Non-additive effects: sex-specific dominance

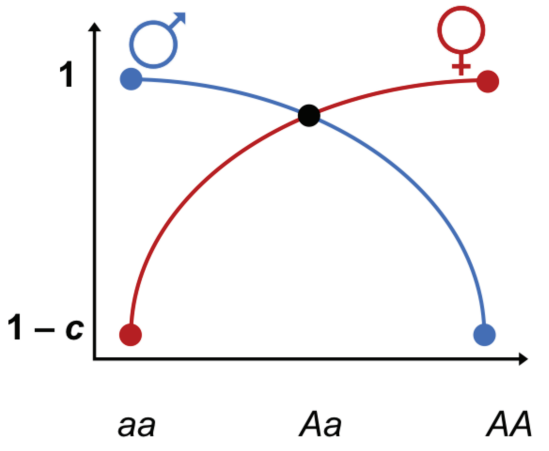

Figure 1: Competitiveness in each sex under different dominance scenarios. $A$ shows male and female phenotypes (competitiveness) according to their genotype. $B-D$ show how male and female competitiveness varies across genotypes depending on allele dominance effects when costs to homozygotes are equal across the sexes $\left(c=c_{\mathrm{m}}=c_{\mathrm{f}}\right.$ ). B shows additive allele effects in both sexes (codominance, $h_{\mathrm{m}}=h_{\mathrm{f}}=0.5$ ). C shows nonadditive effects (incomplete dominance) where the dominant and recessive alleles are the same in both sexes $\left(h_{\mathrm{m}}=1-h_{\mathrm{f}} \neq 0.5\right)$. $D$ shows a specific case of nonadditivity and sex-specific dominance effects $\left(h_{\mathrm{m}} \neq 1-h_{\mathrm{f}}\right)$, known as dominance reversal, whereby the detrimental allele in each sex is recessive $\left(h_{\mathrm{m}}, h_{\mathrm{f}}<0.5\right)$.

in the population of the $A$ allele at an autosomal locus over one generation is

$$
\Delta p(p)=\frac{1}{2} p(1-p)\left(c_{\mathrm{f}} \times s_{\mathrm{f}}(p)-c_{\mathrm{m}} \times s_{\mathrm{m}}(p)\right),
$$

where $p(1-p)$ is the genetic variance in the population, $c_{\mathrm{f}} \times s_{\mathrm{f}}(p)$ captures (positive) selection on $A$ due to its effect in females, and $-c_{\mathrm{m}} \times s_{\mathrm{m}}(p)$ captures (negative) selection on $A$ due to its effect in males (for derivation, see app. A, secs. A.2.1-A.2.5). Selection on females and males, $s_{\mathrm{f}}(p)$ and $s_{\mathrm{m}}(p)$, both depend on allelic frequency $p$ and can be decomposed as the sum of three relevant effects,

$$
\begin{aligned}
s_{\mathrm{f}}(p)= & \underbrace{p+F_{\mathrm{IT}}(1-p)}_{\text {direct effect in } A A}+\underbrace{\left(1-h_{\mathrm{f}}\right)(1-2 p)\left(1-F_{\mathrm{IT}}\right)}_{\text {direct effect in } A a} \\
& -\underbrace{\left(1-m_{\mathrm{f}}\right)^{2}\left[K+2\left(F_{\mathrm{ST}}-K\right)\left(p+\left(1-h_{\mathrm{f}}\right)(1-2 p)\right)\right]}_{\text {kin competition }},
\end{aligned}
$$

$$
\begin{aligned}
s_{\mathrm{m}}(p)= & \underbrace{p+F_{\mathrm{IT}}(1-p)}_{\text {direct effect in } A A}+\underbrace{h_{\mathrm{m}}(1-2 p)\left(1-F_{\mathrm{IT}}\right)}_{\text {direct effect in } A a} \\
& -\underbrace{\left(1-m_{\mathrm{m}}\right)^{2}\left[K+2\left(F_{\mathrm{ST}}-K\right)\left(p+h_{\mathrm{m}}(1-2 p)\right)\right]}_{\text {kin competition }},
\end{aligned}
$$

where $F_{\mathrm{IT}}, F_{\mathrm{ST}}$, and $K$ denote probabilities that different types of genes are identical by descent (IBD) under neutrality (when $c_{\mathrm{f}}=c_{\mathrm{m}}=0$ ), capturing various genetic consequences of limited dispersal. The term $F_{\mathrm{IT}}$ is the probability that the two homologous genes at an autosomal locus of one individual are IBD (which corresponds to the absolute coefficient of inbreeding in the infinite island model; Wright 1922; Caballero 1994; Rousset 2004). Meanwhile, $F_{\text {ST }}$ is the probability that two autosomal genes in two different juveniles of the same sex and group before dispersal are IBD. This measures the degree of genetic differentiation among groups and is proportional to standard relatedness 
coefficients (Rousset 2004). As a result of random mating within groups, these coefficients are equal in male and female juveniles prior to dispersal. After sex-specific dispersal, however, male and female genetic differentiation among groups diverge, becoming $\left(1-m_{\mathrm{m}}\right)^{2} F_{\mathrm{ST}}$ and $\left(1-m_{\mathrm{f}}\right)^{2} F_{\mathrm{ST}}$, respectively (where recall $m_{\mathrm{m}}$ and $m_{\mathrm{f}}$ are sexspecific probabilities of dispersal). Finally, $K$ is the probability that the two homologous genes of a juvenile plus a third gene sampled in another juvenile of the same sex and from the same group are all IBD (also prior to dispersal). As such, $\left(1-m_{\mathcal{u}}\right)^{2} K$ is the probability that among two competing individuals of sex $u$ after dispersal, one is homozygous for a given allele and the other carries at least one IBD copy of that allele.

To understand equation (2) and how limited dispersal influences selection, note first that in a well-mixed and randomly mating population (where $F_{\mathrm{IT}}=F_{\mathrm{ST}}=K=$ $0)$, selection reduces to $s_{\mathrm{f}}(p)=p+\left(1-h_{\mathrm{f}}\right)(1-2 p)$ and $s_{\mathrm{m}}(p)=p+h_{\mathrm{m}}(1-2 p)$ (as found previously; e.g., Mullon et al. 2012, eq. [2]). In these baseline expressions, the term $p$ captures selection on $A$ due to the effects of the allele on the fitness of its bearer in homozygotes, while $\left(1-h_{\mathrm{f}}\right)(1-2 p)$ and $h_{\mathrm{m}}(1-2 p)$ capture selection on $A$ through female and male heterozygotes, respectively. Under limited dispersal, the direct effects of $A$ in homozygotes (labeled "direct effect in $A A$ " in eq. [2]) increase to $p+F_{\mathrm{IT}}(1-p)$ in both sexes. Conversely, the direct effects of $A$ in heterozygotes (labeled "direct effect in $A a$ " in eq. [2] $)$ decrease to $\left(1-h_{\mathrm{f}}\right)(1-2 p)\left(1-F_{\text {IT }}\right)$ in females and to $h_{\mathrm{m}}(1-2 p)\left(1-F_{\mathrm{IT}}\right)$ in males. Selection through homozygotes is therefore more important under limited dispersal. This is because mating within groups leads to inbreeding and therefore a relative excess of homozygotes and a deficit of heterozygotes (according to the inbreeding coefficient $F_{\mathrm{IT}}$ ).

The remaining terms of equation (2) (labeled "kin competition") capture a second effect of limited dispersal: that competing individuals are more likely to carry identical gene copies than randomly sampled individuals. Such kin competition effects increase in both sexes with the probabilities that different competing individuals carry IBD genes $\left(\left(1-m_{\mathrm{f}}\right)^{2} F_{\text {ST }}\right.$ and $\left(1-m_{\mathrm{f}}\right)^{2} K$ in females, and $\left(1-m_{\mathrm{m}}\right)^{2} F_{\mathrm{ST}}$ and $\left(1-m_{\mathrm{m}}\right)^{2} K$ in males). As shown by the negative sign in front of these terms in equation (2), kin competition effects oppose direct selection effects and therefore weaken the selective force favoring the most adaptive allele in each sex. This is because kin competition results in an individual's reproductive success coming at the expense of genetic relatives, thus reducing the strength of selection on competitiveness.

Additive Effects: Selection Favors the Most Dispersive Sex. Equation (2) shows that where dispersal differs between males and females $\left(m_{\mathrm{f}} \neq m_{\mathrm{m}}\right)$, the strength of kin competition differs among the sexes. Sex-specific dispersal therefore biases the intersexual tug-of-war over allele frequency when competition occurs after dispersal (i.e., under hard selection; for soft selection, see eq. [A-35] in app. A, sec. A.2.6). Specifically, the sex that is the most dispersal limited - and therefore experiences the greatest level of kin competition - is under weaker selection for competitiveness. This can be seen more clearly if we consider an additive locus with equally antagonistic allele effects $\left(h_{\mathrm{f}}=h_{\mathrm{m}}=1 / 2\right.$ and $\left.c_{\mathrm{f}}=c_{\mathrm{m}}=c\right)$. In this case, equations (1) and (2) reduce to

$$
\Delta p(p)=c F_{\mathrm{ST}} p(1-p)\left(1-\frac{m_{\mathrm{m}}+m_{\mathrm{f}}}{2}\right)\left(m_{\mathrm{f}}-m_{\mathrm{m}}\right),
$$

whose sign is determined by the sign of $m_{\mathrm{f}}-m_{\mathrm{m}}$ (for $0<$ $p<1$; by contrast, $\Delta p(p)=0$ for all $p$ for an additive locus with equally weak antagonistic effects in well-mixed populations; Fry 2010). This means that according to the sign of $m_{\mathrm{f}}-m_{\mathrm{m}}$, allele $A$ or allele $a$ fixes. Femalebeneficial $A$ fixes when $m_{\mathrm{f}}>m_{\mathrm{m}}$, and male-beneficial $a$ fixes when $m_{\mathrm{f}}<m_{\mathrm{m}}$. This yields a simple rule for the fate of an additive sexually antagonistic allele with symmetric effects: the allele that benefits the most dispersive sex will fix, and this is because the sex that disperses the most suffers less intense kin competition than the other.

Nonadditive Effects: Subdivision Impedes the Maintenance of Sexually Antagonistic Polymorphism. In a wellmixed population, selection can favor the maintenance of sexually antagonistic polymorphism at an autosomal locus when sex-specific dominance is such that the effect of the detrimental allele in each sex is recessive, that is, under dominance reversal (mathematically, reciprocal invasion $-\Delta p^{\prime}(0)>0$ and $\Delta p^{\prime}(1)<0$, where the prime symbol refers to differentiation with respect to $p$-is favored when $h_{\mathrm{f}}<1 / 2$ and $h_{\mathrm{m}}<1 / 2$; fig. $1 D$; Fry 2010; Kidwell et al. 1977, eq. [3]). To investigate the effects of population subdivision on sexually antagonistic polymorphisms with sex-specific dominance, we first calculated the relevant coalescence probabilities $\left(F_{\mathrm{IT}}, F_{\mathrm{ST}}, K\right)$ in equations (1) and (2) in terms of demographic parameters following standard identity-by-descent arguments (e.g., for dioecious subdivided populations, see Ramachandran et al. 2008; Van Cleve et al. 2010; Wang 1997; for derivation, see our app. A, sec. A.2.7). We find that these coalescence probabilities are complicated expressions that depend on sexspecific dispersal $\left(m_{\mathrm{f}}\right.$ and $\left.m_{\mathrm{m}}\right)$ as well as the number of reproducing adults in each group $\left(n_{\mathrm{f}}\right.$ and $\left.n_{\mathrm{m}}\right)$. When dispersal among groups is low and group size is large, they simplify to 


$$
\begin{aligned}
F=F_{\mathrm{IT}}=F_{\mathrm{ST}} & =\frac{1}{1+8\left(M_{\mathrm{m}}(1-r)+M_{\mathrm{f}} r\right)}, \\
K & =\frac{F}{1+4\left(M_{\mathrm{m}}(1-r)+M_{\mathrm{f}} r\right)},
\end{aligned}
$$

where $M_{\mathrm{f}}=n_{\mathrm{f}} m_{\mathrm{f}}$ and $M_{\mathrm{m}}=n_{\mathrm{m}} m_{\mathrm{m}}$ are the expected numbers of female and male immigrants, respectively, in a patch at each generation and $r=n_{\mathrm{m}} /\left(n_{\mathrm{m}}+n_{\mathrm{f}}\right)$ is the proportion of males among adults in a patch (i.e., the adult sex ratio; note that our expression for $F$ in eq. [4] is equivalent to eq. [8] in Ramachandran et al. 2008). Unsurprisingly, equation (4) shows that coalescence becomes more likely when there are fewer immigrants $\left(M_{\mathrm{m}}\right.$ and $M_{\mathrm{f}}$ are small). But equation (4) further tells that coalescence probabilities increase when the adult and immigrant sex ratios are both biased in a similar way (i.e., when $M_{\mathrm{m}}>$ $M_{\mathrm{f}}$ and $r>0.5$ or when $M_{\mathrm{m}}<M_{\mathrm{f}}$ and $r<0.5$; for the exact $F_{\mathrm{ST}}$ where patches are small, see fig. $2 A$ ). This is because such local bottleneck effects on the nondispersing sex increases the coalescence of gene lineages through this sex disproportionately.

Substituting equation (4) (which assumes that patches are large) into equations (1) and (2), we find that selection favors the maintenance of sexually antagonistic polymorphism (i.e., $\Delta p^{\prime}(0)>0$ and $\Delta p^{\prime}(1)<0$ ) when

$\frac{h_{\mathrm{m}}}{1-h_{\mathrm{f}}}+F \frac{1-h_{\mathrm{f}}-h_{\mathrm{m}}}{1-h_{\mathrm{f}}}<\frac{c_{\mathrm{f}}}{c_{\mathrm{m}}}<\frac{1-h_{\mathrm{m}}}{h_{\mathrm{f}}}-F \frac{1-h_{\mathrm{f}}-h_{\mathrm{m}}}{h_{\mathrm{f}}}$.

Where $F=0$, condition equation (5) reduces to the weak selection condition for maintenance of polymor- phism in a well-mixed population (e.g., Fry 2010, eq. [3], under weak selection, i.e., ignoring higher-order selection effects, which using his notation are terms $\mathcal{O}\left(s_{\mathrm{m}}^{2}\right), \mathcal{O}\left(s_{\mathrm{f}}^{2}\right)$, and higher). But as dispersal becomes limited in both sexes (and $F$ increases), equation (5) reveals that the conditions for polymorphism become more stringent (as the lower and upper bounds for $c_{\mathrm{f}} / c_{\mathrm{m}}$ increase and decrease, respectively, with F). To check whether this holds more generally (i.e., for small patches), we analyzed equations (1) and (2) numerically using exact coalescence probabilities (see app. A, sec. A.2.7). Similarly, we find that the parameter conditions favoring polymorphism become increasingly restrictive as dispersal becomes more limited (see the gray region in fig. $3 A$, top), indicating that our conclusions also hold when patches are small (more generally, as highlighted by eqq. [1] and [2], patch size in itself has no direct effect on selection; instead, what matters are genetic associations within and between individuals as summarized by $F$ statistics; see also suppl. fig. 1; suppl. figs. 1, 2 are available online). Together, these analyses show that limited dispersal decreases the scope for the maintenance of sexually antagonistic variation. This is because limited dispersal leads to inbreeding so that selection through heterozygotes, which favors sexually antagonistic polymorphism under dominance reversal, is less relevant than in wellmixed populations.

We also derived the equilibrium allele frequency when polymorphism is favored under limited dispersal (see eq. [A-47] in app. A, sec. A.2.8). In line with equation (5), we find that limited dispersal increases the equilibrium frequency of the allele with the weaker detrimental effect
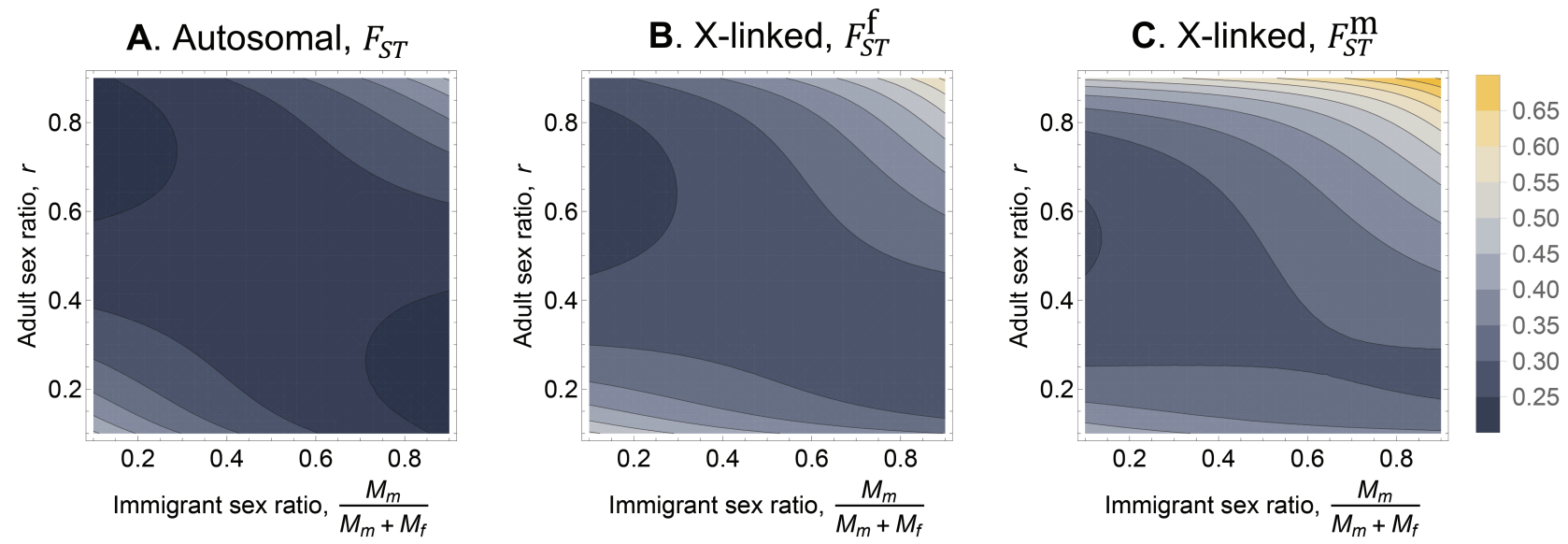

Figure 2: Coalescence probabilities according to adult and immigrant sex ratio. Shown are contour plots of the probability that two neutral genes carried by two randomly sampled group neighbors are identical by descent when these genes are residing on autosomes $(A)$, residing on the $\mathrm{X}$ chromosomes of females $(B)$, and residing on the $\mathrm{X}$ chromosomes of males $(C)$. For calculations, see appendix A, sections A.2.7 and A.3.2. Other parameters: number of immigrants per generation, $M_{\mathrm{m}}+M_{\mathrm{f}}=1$; group size, $n_{\mathrm{m}}+n_{\mathrm{f}}=10$ (although note that group size has little effect on $F$ statistics compared with the average numbers $M_{\mathrm{m}}$ and $M_{\mathrm{f}}$ of immigrants per generation; see eqq. [4] and [7]; see also Rousset 2004). 


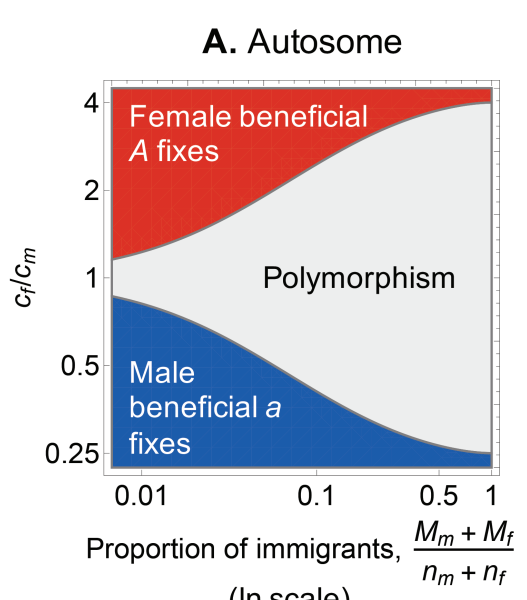

(In scale)

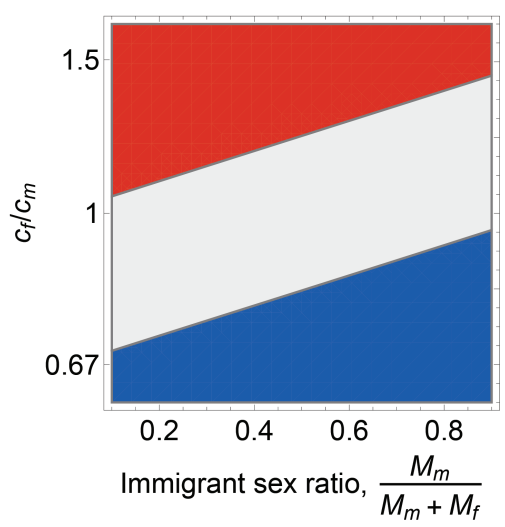

B. X-chromosome
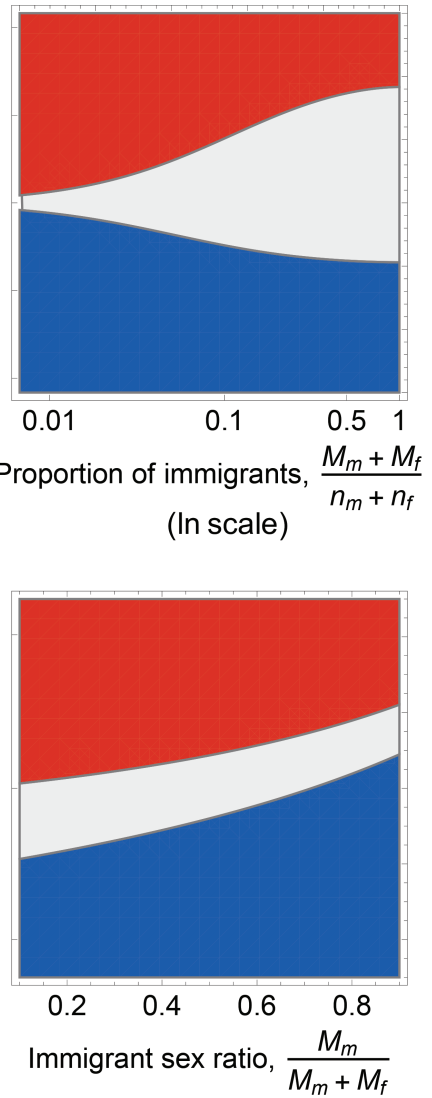

Figure 3: Parameters that favor the maintenance of sexually antagonistic variation. Shown are combinations of parameters that lead to balancing selection (gray, when $\Delta p^{\prime}(0)>0$ and $\Delta p^{\prime}(1)<0$ ), positive selection for allele $A$ (red, when $\Delta p^{\prime}(p)>0$ for all $p$ ), and positive selection for allele $a$ (blue, when $\Delta p^{\prime}(p)<0$ for all $p$ ) at an autosomal locus ( $A$; computed from eqq. [1] and [2] with exact coalescence probabilities; for calculations of these probabilities, see app. A, sec. A.2.7) and an X-linked locus (B; computed from eqq. [A-55]-[A-57] with exact coalescence probabilities; for calculations, see app. A, sec. A.3.2). Top, selection according to the ratio of homozygotic effects in females and males $\left(c_{\mathrm{m}} / c_{\mathrm{f}}\right)$ and the expected proportion of immigrants in each patch at each generation, $\left(M_{\mathrm{m}}+M_{\mathrm{f}}\right) /\left(n_{\mathrm{m}}+n_{\mathrm{f}}\right)$ (on a natural log scale; for linear scale, see suppl. fig. 1; other parameters: $h_{\mathrm{f}}=h_{\mathrm{m}}=0.2, M_{\mathrm{m}}=M_{\mathrm{f}}, n_{\mathrm{m}}=n_{\mathrm{f}}=5$; for the effect of $F$ statistic in large groups, see suppl. fig. 1). Bottom, selection according to the ratio of homozygotic effects in females and males $\left(c_{\mathrm{m}} / c_{\mathrm{f}}\right)$ and the expected proportion of males among immigrants in each patch at each generation $\left(M_{\mathrm{m}} /\left(M_{\mathrm{m}}+M_{\mathrm{f}}\right)\right.$; other parameters:, $n_{\mathrm{m}}=n_{\mathrm{f}}=5, h_{\mathrm{f}}=h_{\mathrm{m}}=0.2, M_{\mathrm{m}}+$ $\left.M_{\mathrm{f}}=0.1\right)$.

in homozygotes, irrespective of dominance (e.g., if $c_{\mathrm{m}}<$ $c_{\mathrm{f}}$, the equilibrium frequency of the male-detrimental allele $A$ increases with limited dispersal). In other words, where there is polymorphism, inbreeding leads selection to disproportionately direct equilibrium allele frequencies based on selective effects in homozygotes rather than heterozygotes.

Nonadditive Effects: Sex-Biased Dispersal Changes the Nature of Sexually Antagonistic Polymorphism. Analyzing equation (5) further reveals that the nature of sexually antagonistic alleles maintained by balancing selection depends on immigrant sex ratio (fig. $3 A$, bottom). Specif- ically, as dispersal becomes biased toward one sex, polymorphism tends to be favored when the cost of the detrimental allele to the philopatric sex is greater than the cost of the detrimental allele to the dispersive sex (e.g., where dispersal is male biased, $M_{\mathrm{m}} /\left(M_{\mathrm{m}}+M_{\mathrm{f}}\right)>0.5$, polymorphism is more likely when $c_{\mathrm{f}}>c_{\mathrm{m}}$; see the gray region in fig. $3 A$, bottom). Accordingly, sex-biased dispersal also relaxes the condition for fixation of the allele beneficial to the dispersing sex and constrains the condition favoring fixation of the allele beneficial to the philopatric one (e.g., male-biased dispersal increases the parameter space for fixation of the male-beneficial allele but decreases parameter space for fixation of the female-beneficial allele; 
see the blue and red regions in fig. $3 A$, bottom). In line with our analysis of the additive case (eq. [3]), these effects occur because sex-specific dispersal leads the philopatric sex to experience stronger kin competition, weakening the selective advantage of the allele favoring competitiveness in that sex relative to the other. As a result, sex differences in dispersal proclivity lead to different types (i.e., competitiveness effects) of alleles being maintained under balancing selection, thereby influencing the nature of sexually antagonistic polymorphism.

Effects of Limited Dispersal Are Similar at X-linked and Autosomal Loci. We also derived the frequency change of allele $A$ when linked to an $\mathrm{X}$ chromosome (our results also apply to Z linkage in ZW species; for derivations, see app. A, secs. A.3.1 and A.3.2). Our analysis of the resulting allelic dynamics, which can be found in appen$\operatorname{dix}$ A, section A.3.3, shows that the effects of limited dispersal on X-linked sexual antagonism are broadly the same as those for autosomes. Specifically, inbreeding also leads to more constrained polymorphism conditions on the $\mathrm{X}$ chromosome (fig. 3B, top; for a recent discussion of inbreeding effects on X-linked sexual antagonism, see Hitchcock and Gardner 2020), and sex-specific dispersal also favors adaptation in the more dispersive sex at the expense of the other (so that polymorphism tends to be favored when the cost of the detrimental allele is greater in the philopatric sex; fig. $3 B$, bottom).

There are nevertheless some discrepancies between autosomal and $\mathrm{X}$-linked positions as a result of the fact that males only carry one copy of the X chromosome. First, the difference in copy number between the sexes means that male reproductive value for X-linked genes is half that of females (because on average males transmit half as many $\mathrm{X}$ copies to their offspring as females). As a result, selection via female competition is twice as important as that via male competition on the $\mathrm{X}$ chromosome. The consequences of this can be most clearly seen by considering an X-linked additive locus $\left(h_{\mathrm{f}}=1 / 2\right)$ with equal allelic effects in males and females $\left(c_{\mathrm{f}}=c_{\mathrm{m}}=c\right)$. We show in appendix A, section A.3.3, that at such a locus, the change in $p$ per generation is given by

$$
\Delta p(p)=c F_{\mathrm{X}} p(1-p)\left(2 m_{\mathrm{f}}\left(2-m_{\mathrm{f}}\right)-m_{\mathrm{m}}\left(2-m_{\mathrm{m}}\right)\right),
$$

where

$$
F_{\mathrm{X}}=\frac{1+r}{1+r+6\left(M_{\mathrm{m}}(1-r)+2 M_{\mathrm{f}} r\right)}
$$

is the two-way coalescence probability for the $\mathrm{X}$ chromosome (i.e., the probability that two neutral genes sampled from the same sex in the same group before dispersal are
IBD in the limit of low dispersal and large group size; for derivation of $F_{X}$, see app. A, sec. A.3.2; note that eq. [7] is equivalent to eq. [9] in Ramachandran et al. 2008). Equation (6) shows that under weak additive effects, selection favors the fixation of one allele or the other depending on sex-specific dispersal, as on autosomes. But unlike on autosomes, for the male-beneficial allele to be favored, males must be twice as likely to disperse than females (i.e., $\Delta p(p)<0$ for all $0<p<1$ when $m_{\mathrm{m}}>2 m_{\mathrm{f}}$; by contrast, this occurs when $m_{\mathrm{m}}>m_{\mathrm{f}}$ on autosomes; see eq. [3]).

Comparing the probabilities that autosomal and $\mathrm{X}$ linked genes are IBD (eq. [4] vs. eq. [7]) reveals a second effect of male hemizygosity: that X-linked genes in individuals from the same group tend to coalesce faster than autosomal genes, with the exception of where sex and immigrant ratios are both strongly female biased (specifically, $F>F_{\mathrm{X}}$ only when $r<0.5$ and $M_{\mathrm{m}} /\left(M_{\mathrm{m}}+M_{\mathrm{f}}\right)<$ $2 r(2 r-1) /\left(8 r^{2}-5 r-1\right)$; fig. $2 A$, bottom left corner $)$. This is because there are always fewer $\mathrm{X}$ copies than autosomes in a group. Furthermore, as for autosomes, the probability of X-linked coalescence increases when the adult and immigrant sex ratios are biased in a similar manner; however, this occurs to a greater extent on the $\mathrm{X}$ when both ratios are biased toward males (for exact probabilities, see fig. $2 B, 2 C$ ). As a consequence, polymorphism conditions tend to be more restrictive on the $\mathrm{X}$ chromosome than on autosomes with dispersal limitation (fig. $3 A, 3 B$, top), especially when immigrants are more likely to be males (fig. $3 B$, bottom).

\section{The Interplay between Sexually Antagonistic Selection and Genetic Drift under Limited Dispersal}

By assuming an infinite number of groups, our analyses so far have ignored the influence of genetic drift, which is a pervasive evolutionary force in finite populations (Charlesworth and Charlesworth 2010) and thought to be especially relevant for intralocus sexual conflict (Connallon and Clark 2012; Mullon et al. 2012). Predicting how sexually antagonistic variation is maintained under the joint effects of selection and genetic drift in subdivided populations, however, is not straightforward. This is because dispersal limitation influences both balancing selection and genetic drift with conflicting implications for polymorphism. On the one hand, because limited dispersal leads to kin competition and inbreeding, it weakens the strength of balancing selection relative to genetic drift. On the other hand, genetic isolation between patches increases the effective size $\left(N_{\mathrm{e}}\right)$ of a population and therefore reduces the strength of genetic drift relative to selection (in the absence of patch extinction; Caballero 1994; Wang and Caballero 1999). 
A

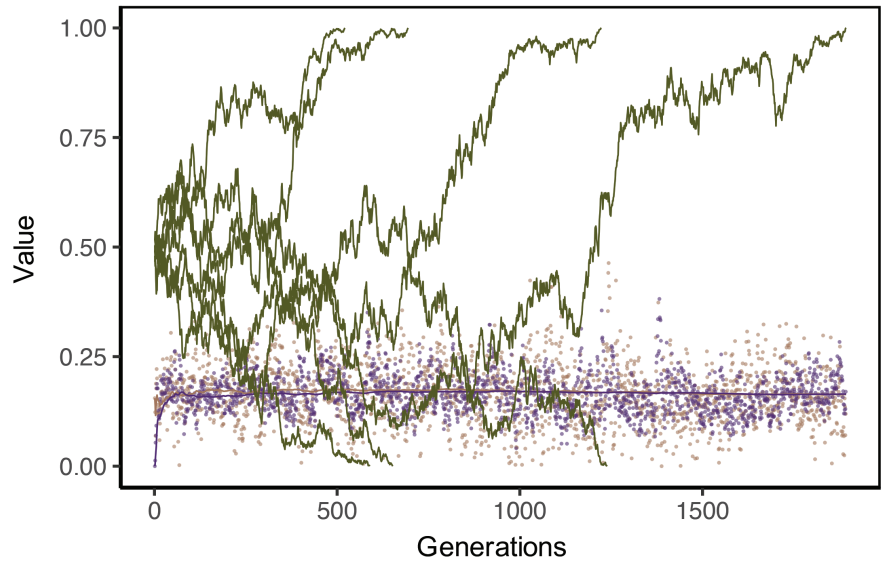

B

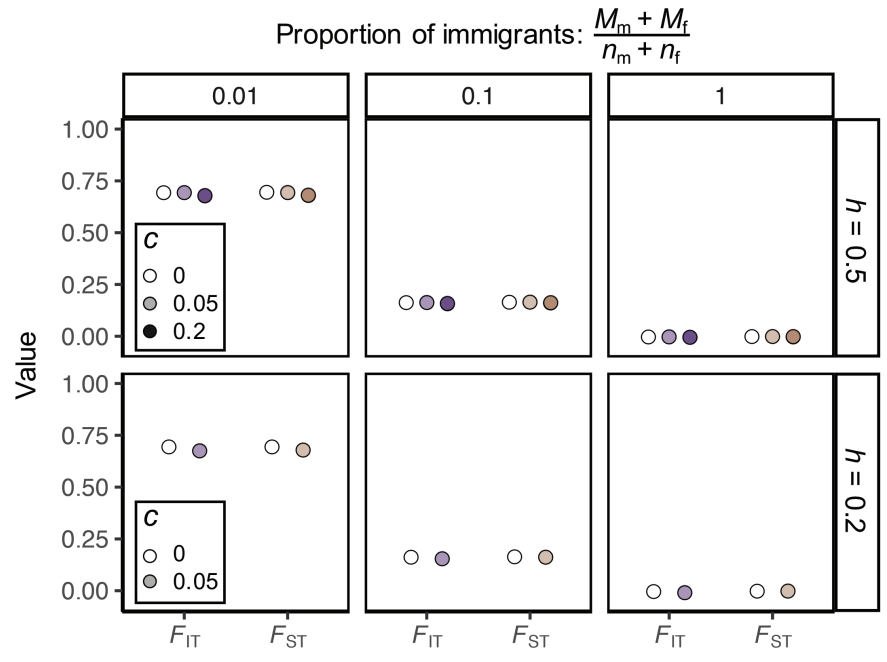

C

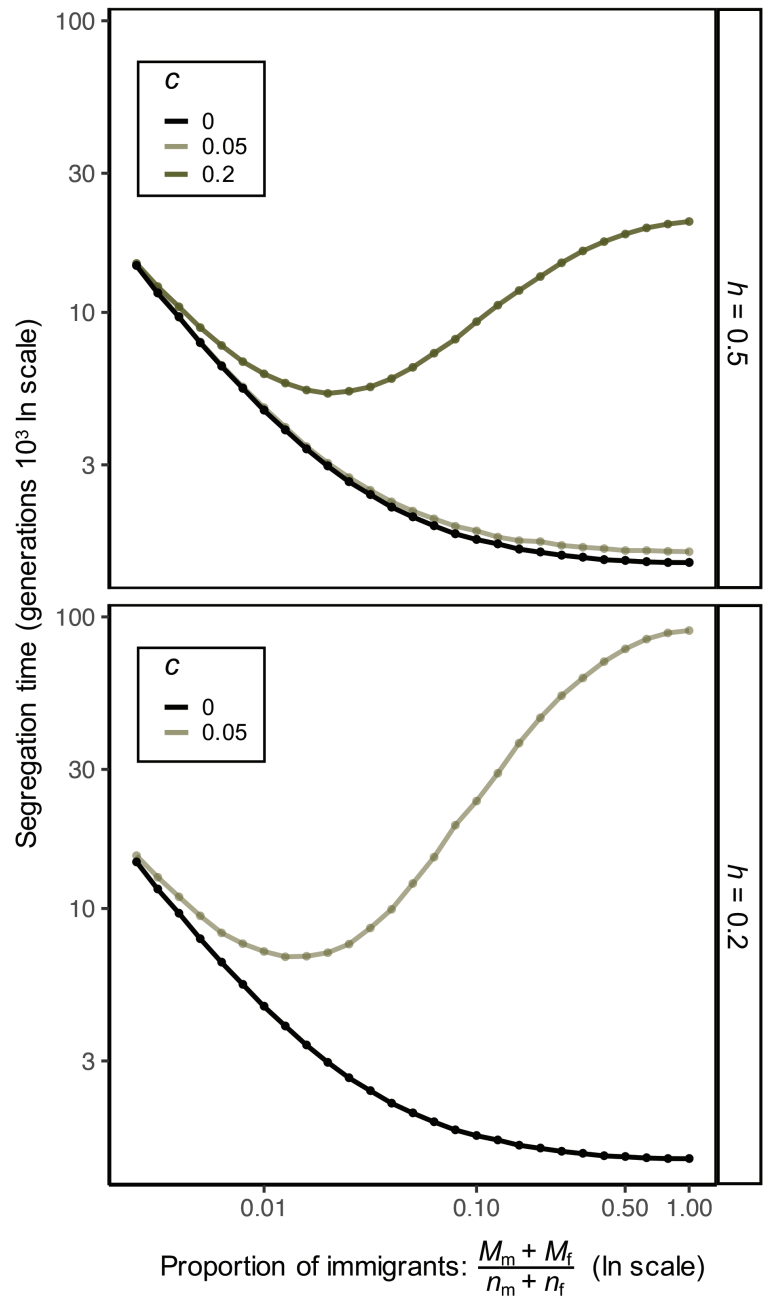

Figure 4: Allele segregation time and genetic variation in individual-based simulations. $A$ shows example simulation runs under neutrality $\left(c=c_{\mathrm{m}}=c_{\mathrm{f}}=0\right)$ and limited dispersal (when the expected proportion of immigrants per group per generation $\left(M_{\mathrm{m}}+M_{\mathrm{f}}\right) /\left(n_{\mathrm{m}}+\right.$ $\left.n_{\mathrm{f}}\right)=0.1$ ). Green trajectories show the frequency of allele $A$ for different replicate runs, where the time taken for $A$ to fix or be lost represents "segregation time" for that simulation. Purple and orange circles show $F_{\mathrm{IT}}$ and $F_{\mathrm{ST}}$, respectively, at each generation during a simulation, while purple and orange lines represent time-averaged values for these quantities (for details on how $F_{\mathrm{IT}}$ and $F_{\mathrm{ST}}$ are calculated, see eqq. [B-1] and [B-2] in app. B). B shows time-averaged $F_{\mathrm{IT}}$ and $F_{\mathrm{ST}}$ averaged across all $(10,000)$ replicate runs under different levels of limited dispersal (the number at the top of each column panel gives the expected proportion of immigrants in each group), dominance effects (row panels indicate $h=h_{\mathrm{f}}=h_{\mathrm{m}}$ ), and strengths of sexually antagonistic selection (white circles represent neutrality $c=0$; light- and darkcolored circles represent $c=0.05$ and $c=0.2$, respectively). Note that because the $F_{\mathrm{ST}}$ and $F_{\mathrm{IT}}$ estimates for each replicate are already averaged across generations in a simulation, these statistics are highly consistent between simulation runs (standard deviation of order $10^{-2}$ or lower). $C$ shows segregation time averaged across replicate runs as a function of the total fraction of immigrants (which is on a natural log scale; for linear scale, see suppl. fig. 2) and selection strength (black line for neutrality, solid light and dark green lines for $c=0.05$ and $c=0.2$, respectively; for $c=0.01$, see suppl. fig. 2). Row panels again show different dominance effects. Circles refer to parameter combinations (proportion of immigrants and strength of selection) used in simulations. Other parameter values for simulations: $n_{\mathrm{m}}=n_{\mathrm{f}}=5$ (for more details on the simulation procedure, see app. B). 
A Simulation Approach. To investigate the conflicting effects of limited dispersal on the maintenance of polymorphism at sexually antagonistic loci through selection and genetic drift, we used individual-based simulations to track the dynamics of sexually antagonistic alleles at a diallelic autosomal locus in a finite subdivided population (for full simulation procedure with SLiM 3.3, see app. B; code is provided in a zip file, available online; Haller and Messer 2019). ${ }^{1}$ Our simulations follow a population of 50 groups each containing equal numbers of male and female adults (for computational tractability, $n_{\mathrm{m}}=n_{\mathrm{f}}=5$; for additional simulations where $n_{\mathrm{m}}=$ $n_{\mathrm{f}}=10$, which produced results that were qualitatively equivalent to those in this section, see suppl. fig. 2).

At the beginning of a generation, each female in a group produces a Poisson-distributed number of eggs (mean $k=20$ ). Males are then sampled at random with replacement within groups to fertilize each egg and produce a zygote. Zygotes are assigned male or female identity with equal probability (i.e., sex ratio is unbiased at birth). Male and female zygotes disperse to another randomly selected group with sex-specific probability $m_{\mathrm{m}}$ and $m_{\mathrm{f}}$ or stay in their natal group otherwise. Finally, $n_{\mathrm{m}}$ male and $n_{\mathrm{f}}$ female zygotes are sampled without replacement in each patch to reach adulthood, with the probability of a zygote being sampled weighted by the competitiveness of its genotype (according to fig. $1 A$ ).

We ran simulations for a number of dispersal regimes $\left(m_{\mathrm{m}}, m_{\mathrm{f}}\right)$ and allelic effects $\left(c_{\mathrm{m}}, c_{\mathrm{f}}\right)$. We also considered additive $\left(h_{\mathrm{m}}=h_{\mathrm{f}}=0.5\right)$ and dominance reversal $\left(h_{\mathrm{m}}=\right.$ $\left.h_{\mathrm{f}}=0.2\right)$ scenarios. Simulations were started with equal frequencies of male- and female-beneficial alleles in the population ( $p=0.5$ at Hardy-Weinberg equilibrium) and then left to run until one allele is fixed and the other is lost (for examples of runs, see the green trajectories in fig. 4A). We ran 10,000 replicates for each parameter combination. For each simulation replicate, we tracked segregation time (number of generations until loss of an allele), the final frequency of the female-beneficial allele $A$ ( 0 or 1 ), and the $F_{\mathrm{IT}}$ and $F_{\mathrm{ST}}$ statistics in adults (which were time averaged across generations in a replicate; for an example, see the purple and orange circles and lines in fig. $4 A$; for details, see app. B).

Strong Sexual Antagonism, Dominance Reversal, and Frequent Dispersal Prolong Polymorphism in Finite Populations. We first consider the case where allelic effects are equal in both sexes $\left(c=c_{\mathrm{m}}=c_{\mathrm{f}}\right)$ and where dispersal is unbiased $\left(m_{\mathrm{m}}=m_{\mathrm{f}}\right)$. In line with equation (4), we observe that as dispersal probability decreases, inbreeding,

1. Code that appears in The American Naturalist is provided as a convenience to readers. It has not necessarily been tested as part of peer review.
$F_{\mathrm{IT}}$, and genetic differentiation among groups, $F_{\mathrm{ST}}$, both increase (irrespective of the strength of selection and dominance effects; fig. $4 B$ ). In other words, as dispersal becomes limited we see a reduction in genetic variation within individuals and within groups as well as an increase in genetic variation between groups. Such conditions are known to increase effective population size (Wang and Caballero 1999). Accordingly, limited dispersal prolongs the maintenance of neutral genetic variation in our simulations $(c=0$; black line in fig. 4C).

For sexually antagonistic loci, the effect of dispersal on segregation time depends on whether sexual antagonism leads to balancing selection. Where alleles have weak additive $\left(h_{\mathrm{m}}=h_{\mathrm{f}}=0.5\right)$ effects that are symmetric across the sexes $\left(c_{\mathrm{m}}=c_{\mathrm{f}}=c \ll 1\right)$, selection is effectively neutral in the absence of sex-specific dispersal (eq. [3]). In this case, the impact of limited dispersal on segregation time is largely similar to its effect on a neutral locus, with variation maintained for longer when dispersal is limited (compare black and pale green in fig. $4 C$, top). By contrast, when selection is balancing, either as a result of dominance reversal $\left(h_{\mathrm{m}}<0.5, h_{\mathrm{f}}<0.5\right.$; eq. [5]) or strong selection (i.e., large $c_{\mathrm{m}}=c_{\mathrm{f}}=c$; for a well-mixed population, see, e.g., Fry 2010), we observe a quadratic relationship between segregation time and dispersal, with polymorphism maintained for longer when dispersal is either very strong or very weak (dark green curve in fig. $4 C$, top, and light green curve in fig. $4 C$, bottom). Thus, the balance between the opposing effects of population genetic structure on polymorphism, increasing $N_{\mathrm{e}}$ on the one hand and decreasing the strength of selection on the other, varies with the degree to which total dispersal is limited.

Sex-Specific Costs and Dispersal Interact to Modulate Segregation Time. Finally, we consider the segregation of sexually antagonistic alleles where the allelic cost $\left(c_{\mathrm{f}} / c_{\mathrm{m}}\right)$ and immigrant $\left(M_{\mathrm{m}} /\left(M_{\mathrm{m}}+M_{\mathrm{f}}\right)\right)$ ratios can be biased, representing sex differences in selection strength and dispersal proclivity, respectively. We assume dominance reversal $\left(h_{\mathrm{m}}=h_{\mathrm{f}}=0.2\right)$, weak selection $\left(c_{\mathrm{m}}+c_{\mathrm{f}}=0.1\right)$, and a fixed total proportion of immigrants (either $\left(M_{\mathrm{m}}+M_{\mathrm{f}}\right) /\left(n_{\mathrm{m}}+n_{\mathrm{f}}\right)=0.1$ for moderate dispersal limitation or $\left(M_{\mathrm{m}}+M_{\mathrm{f}}\right) /\left(n_{\mathrm{m}}+n_{\mathrm{f}}\right)=0.01$ for strong dispersal limitation).

To understand the results of these simulations, it is useful to recall that sex-specific allele costs and dispersal together determine whether sexual antagonism generates balancing selection (fig. $3 \mathrm{~A}$, bottom) as well as the equilibrium allele frequency, $p^{*}$, favored by such selection (fig. 5A; see also app. A, sec. A.2.8). When the cost and immigrant ratios are such that selection is balancing (so $0<p^{*}<1$; fig. $5 A$ ), our simulations show that polymorphism 


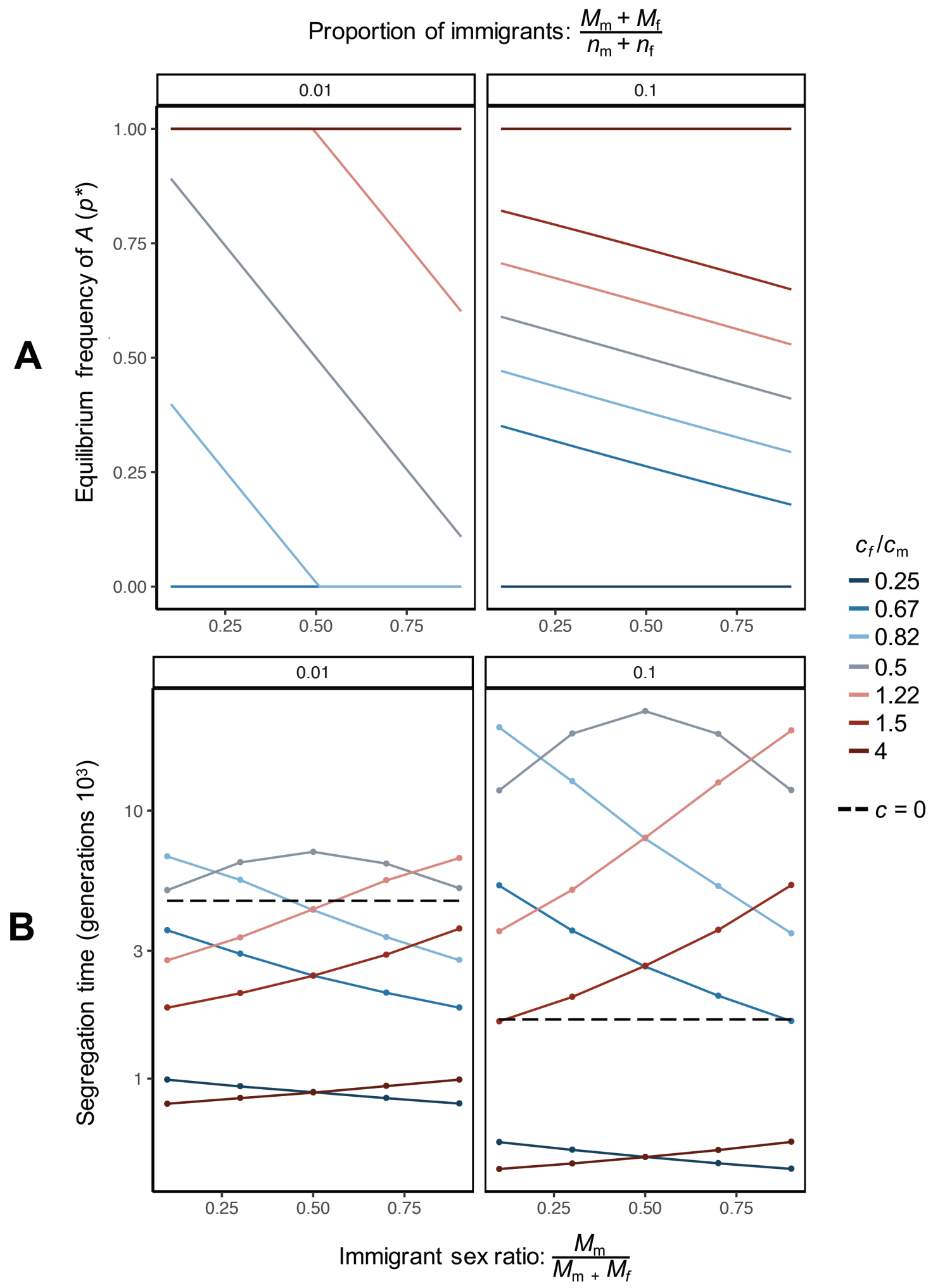

Figure 5: Fate of sexually antagonistic alleles according to sex-specific competitiveness and dispersal effects. Shown are effects of selection and drift on sexually antagonistic alleles when allele cost $\left(c_{\mathrm{f}} / c_{\mathrm{m}}\right)$ and immigrant $\left(M_{\mathrm{m}} /\left(M_{\mathrm{m}}+M_{\mathrm{f}}\right)\right)$ sex ratios are varied. Blue and red lines represent male-biased $\left(c_{\mathrm{f}}<c_{\mathrm{m}}\right)$ and female-biased $\left(c_{\mathrm{f}}>c_{\mathrm{m}}\right)$ competitiveness effects, respectively, while gray lines represent equal costs in 
is long-lived relative to neutral variation (gray, light blue, and light red lines in fig. 5B). In particular, segregation time is greatest when $p^{*}$ is close to 0.5 (i.e., furthest from the fixation boundaries; fig. $5 A, 5 B$ ), in agreement with analyses of finite panmictic populations (Connallon and Clark 2012; Mullon et al. 2012). However, the importance of these selective effects diminishes under strong dispersal limitation. Specifically, segregation time decreases relative to neutral variation and is less sensitive to $p^{*}$ when dispersal is strongly rather than moderately limited (compare left and right panels in fig. 5B). As before, this is because the strength of sexually antagonistic selection decreases relative to drift as dispersal become severely limited and kin competition increases.

Where the cost and immigrant ratios produce directional selection (i.e., selection favors $p=0$ or $p=1$; fig. $5 A$ ), polymorphism is lost more rapidly at a sexually antagonistic locus than at a neutral locus (dark blue and light red lines in fig. 5B), with segregation time lowest when selection strength is most asymmetrical between the sexes and when the dispersing sex is under the more intense selection. This is because these conditions provide the strongest directional selection and thus most rapid fixation of the favored allele. Finally, contrary to where selection is balancing, strongly limited dispersal leads to an increase in segregation time for alleles under directional selection (left vs. right panels in fig. $5 B$ ), as in this case heightened kin competition reduces the efficacy with which such selection purges variation.

\section{Discussion}

Our analyses indicate that limited dispersal can significantly impact evolution at loci under sexual conflict, altering both the nature and the scope of sexually antagonistic variation through three main pathways. First, limited dispersal leads to kin competition, as individuals of the same group are more likely to carry identical alleles than random individuals. Such competition between relatives weakens the strength of selection on competitive traits and hence of sexually antagonistic balancing selection. In addition, where dispersal is sex specific, the strength of kin competition is asymmetric between the sexes and consequently biases the intersexual tug-of-war over allele frequencies in favor of the more dispersive sex (fig. 3, bottom). Second, limited dispersal causes inbreeding and thus reduced heterozygosity. This in turn limits the capacity of dominance reversal to promote polymorphism at sexually antagonistic loci (fig. 3, top). Third, in finite populations, limited dispersal simultaneously reduces both the strength of genetic drift and the strength of sexually antagonistic selection. The implications of this for the maintenance of polymorphism in smaller populations depend on the strength of sexual antagonism, with limited dispersal promoting long-term polymorphism when balancing selection is weak and impeding it when balancing selection is strong (fig. 4C). Our analyses indicate that these various effects of limited dispersal on the segregation of sexually antagonistic alleles are most relevant in subdivided populations characterized by $F_{\mathrm{ST}}$ values of order $10^{-1}$ or higher. Such values are consistent with many $F_{\mathrm{ST}}$ estimates for natural populations (e.g., mammals [Cegelski et al. 2003; Hammond et al. 2006], birds [Woxvold et al. 2006; Harrison et al. 2014], reptiles [Berry et al. 2005; Böhme et al. 2007], and amphibians [Canestrelli et al. 2007; Wang et al. 2020]; for more general comments, see Barton 2001, p. 334; Hartl and Clark 2007, p. 302; Charlesworth and Charlesworth 2010, p. 310). These observations suggest that our model may be relevant for a wide range of taxa.

Our results lead to several predictions for interspecific variation in the distribution of sexually antagonistic alleles. First, in populations with sex-specific dispersal, we expect that sexually antagonistic polymorphism is characterized by greater antagonistic costs - and thus greater fitness variation attributable to sexual antagonism - in the nondispersing sex compared with the dispersing one (e.g., when males disperse more, polymorphism is expected at loci with $c_{\mathrm{f}}>c_{\mathrm{m}}$ ). Although we are not aware of any empirical work explicitly investigating this, there is potentially relevant data from pedigree studies. In the yellowpine chipmunk (Tamias amoenus), a species that shows stronger kinship between neighboring females than males (implying male-biased dispersal; Dobson 1982; SchulteHostedde et al. 2001), females are under directional selection for increased body size while males are not (SchulteHostedde et al. 2002). This suggests that in this species males are closer to their fitness optimum (so that alleles deviating from this optimum incur a small $c_{\mathrm{m}}$ in males)

\footnotetext{
both sexes $\left(c_{\mathrm{f}}=c_{\mathrm{m}}\right)$. Left and right panels indicate different levels of dispersal limitation (left, strong limitation with average proportion of immigrants per generation per group, $\left(M_{\mathrm{m}}+M_{\mathrm{f}}\right) /\left(n_{\mathrm{m}}+n_{\mathrm{f}}\right)=0.01$; right, weak limitation with $\left.\left(M_{\mathrm{m}}+M_{\mathrm{f}}\right) /\left(n_{\mathrm{m}}+n_{\mathrm{f}}\right)=0.1\right)$. A, Equilibrium frequency of allele $A$ under sexually antagonistic selection alone (i.e., assuming an infinite number of patches), calculated numerically by plugging exact coalescence probabilities (see app. A, sec. A.2.7) into equations (1) and (2) and solving $\Delta p\left(p^{*}\right)=0$ for $p^{*}$ (where selection is directional, we set $p^{*}=0$ or $p^{*}=1$ depending on whether selection favors loss or fixation of $A$, respectively). Other parameters: $h_{\mathrm{f}}=h_{\mathrm{m}}=0.2, n_{\mathrm{m}}=n_{\mathrm{f}}=5 . \mathrm{B}$, Segregation time in individual-based simulations averaged across all replicate runs. Circles refer to parameter (cost and immigrant ratio) values used in simulations (for details, see app. B). Other parameters: $h_{\mathrm{f}}=h_{\mathrm{m}}=0.2, n_{\mathrm{m}}=n_{\mathrm{f}}=5$, $c_{\mathrm{f}}+c_{\mathrm{m}}=0.1$. The dashed black line shows the neutral case $\left(c=c_{\mathrm{m}}=c_{\mathrm{f}}=0\right)$.
} 
than females (so that genetic variation for body size in females cause a large $c_{\mathrm{f}}$ ). In the great reed warbler (Acrocephalus arundinaceus), which shows female-biased dispersal (Hansson et al. 2003), sexual antagonism has been described over wing length (Tarka et al. 2014). In line with our prediction, males are under stronger selection for wing length than females and show larger additive genetic variance for fitness (Tarka et al. 2014, tables 1 and 2). However, the authors of this study also report a negative correlation between a male's wing length and the fitness of his female relatives (Tarka et al. 2014, table 3) but not the reverse (a correlation between female wing length and the fitness of male relatives), leading them to suggest that alleles beneficial for males are in fact costlier to females. These conflicting results highlight the difficulty in interpreting the strength of sexually antagonistic allele effects from quantitative genetics studies. More generally, further work is needed to discern the extent to which these observations reflect wider trends, and we suggest that future studies consider the effect of dispersal regimes and mating systems on relatedness when formulating hypotheses on sexually antagonistic variation.

Second, we expect long-term sexually antagonistic polymorphism to accrue less, relative to neutral variation, in dispersal-limited populations than in well-mixed populations. Furthermore, in dispersal-limited species with small total population size (e.g., small fragmented populations), we expect more sexually antagonistic variation than neutral variation when selection and dispersal are both strong. Broadly speaking, we therefore expect different levels of sexually antagonistic variation between species according to population genetic structure. However, because of the close link between dispersal, mating system, and the strength of sex-specific selection (Hamilton 1967; Greenwood 1980; Shields 1987; Perrin and Mazalov 2000; Li and Holman 2018), taxa with contrasting spatial structures may also show differences in the intensity of intralocus conflict, confounding interspecific patterns in sexually antagonistic variation. Instead, a more fine-scale test would be provided by a species with multiple populations of differing spatial structure but similar levels of sexual antagonism. One such potential study system is the Atlantic salmon (Salmo salar), a species lacking sex chromosomes in which quantitative trait locus analyses have identified a polymorphic sexually antagonistic locus that is maintained through sex-specific dominance (fig. $1 D$; Barson et al. 2015). Different salmon populations exhibit different levels of structure, with Scandinavian populations showing strong subdivision (Vähä et al. 2007, 2008) but North American populations appearing relatively panmictic (Wellband et al. 2019). Under the assumption that both salmon populations experience similar patterns of sexually antagonistic selection, this species would be well suited to test the effects of spatial subdivision on the amount of sexually antagonistic variation maintained in a population.

Another, more broad-brush prediction from our results arises from the observation that sex-specific selection over reproductive success tends to be stronger in males (Janicke et al. 2016; Singh and Punzalan 2018). In the context of our study, stronger male selection should cause sexually antagonistic alleles to have greater costs in males than in females (i.e., $c_{\mathrm{m}}>c_{\mathrm{f}}$ in our notation). Where this is true, our results suggest that sexually antagonistic polymorphism is more likely in taxa showing male philopatry (i.e., female-biased dispersal), as heightened male kin competition cancels out the effects of stronger selection for male reproductive success, potentially leading to balancing selection (fig. 3, bottom). Conversely, in taxa where dispersal is predominantly male biased, the combined effects of sexual antagonism and kin competition in females should favor the fixation of malebeneficial alleles, leading to less sexually antagonistic variation and male adaptation at the expense of females.

In addition to being useful for understanding betweenspecies and between-population patterns of sexually antagonistic variation, our results also have implications for variation within genomes. Much debate in studies of sexual antagonism has centered around the likelihood of different genomic regions accumulating sexually antagonistic polymorphisms, in particular whether such variation should be more common on the $\mathrm{X}$ chromosome or autosomes (Rice 1984; Gibson et al. 2002; Gavrilets and Rice 2006; Fry 2010; Otto et al. 2011; Jordan and Charlesworth 2012; Mullon et al. 2012; Ruzicka and Connallon 2020). Previous results have pointed out potential disparities between the $\mathrm{X}$ chromosome and autosomes in their proclivity to harbor sexually antagonistic variation due to male hemizygosity for the $\mathrm{X}$, which leads to differences in selection (i.e., dominance effects act only through females for X-linked genes; Rice 1984; Gavrilets and Rice 2006; Fry 2010; Jordan and Charlesworth 2012) and to differences in the strength of genetic drift (i.e., stronger genetic drift on the $\mathrm{X}$ owing to fewer copies of $\mathrm{X}$ chromosomes than autosomes; Charlesworth et al. 1987; Vicoso and Charlesworth 2009; Mullon et al. 2012). Our findings indicate that population subdivision influences both of these differences. First, limited dispersal diminishes the importance of selection on heterozygotes and thus dominance effects in driving differences in variation between $\mathrm{X}$-linked and autosomal locations (fig. 3). Second, sexspecific dispersal and local demography alter $F_{\mathrm{ST}}$ for Xlinked and autosomally linked genes in different ways (fig. 2) and hence influence the effective population size of the $\mathrm{X}$ chromosome relative to an autosome. In particular, the X-linked and autosomal effective population 
sizes converge if the adult sex ratio within patches is sufficiently female biased under limited dispersal (when $r \approx 0.15$; see Ramachandran et al. 2008, p. 296). Taken together, these results suggest that conditions under which the $\mathrm{X}$ chromosomes and autosomes differ in their propensity to retain sexually antagonistic alleles are less straightforward than predicted by panmictic models. Thus, limited dispersal may help explain the current patchy empirical support for either chromosome region harboring disproportionate levels of sexually antagonistic variation (e.g., Gibson et al. 2002; Lucotte et al. 2016; Grieshop and Arnqvist 2018; Ruzicka et al. 2019).

Our analysis highlights how limited dispersal impacts sexual antagonism by introducing genetic correlations within individuals and groups (via $F_{\mathrm{IT}}$ and $F_{\mathrm{ST}}$ ), skewing genotype frequencies away from Hardy-Weinberg proportions. Alongside limited dispersal, other factors can distort allele distributions away from Hardy-Weinberg equilibrium and thus influence selection on sexually antagonistic variation. In fact, previous theoretical studies have shown that by increasing and decreasing the level of inbreeding, respectively, self-fertilization (Jordan and Connallon 2014; Tazzyman and Abbott 2015) and assortative mating for fitness (Arnqvist 2011; Kasimatis et al. 2019; Hitchcock and Gardner 2020) affect the potential for sexually antagonistic polymorphism (for assortative mating for genotype, see Kasimatis et al. 2019). Meanwhile, $F_{\mathrm{ST}}$ can be influenced by life-history traits other than dispersal, such as iteroparity (which increases genetic relatedness within groups [Taylor and Irwin 2000]). Interestingly, sex asymmetries in relatedness also arise in systems where the sexes differ in the extent to which they are itero- or semelparous (e.g., one sex is long-lived while the other is short-lived [Van Cleve et al. 2010]), suggesting that sex differences in life-history strategies may have similar consequences for sexual antagonism as sexspecific dispersal.

Although our study was framed in the context of sexual antagonism, we also expect population subdivision to have implications for other forms of antagonistic evolution, such as antagonistic pleiotropy (where alleles have antagonistic effects on different fitness components; e.g., Williams 1957; Rose 1982; Kirkwood and Rose 1991; Curtsinger et al. 1994; Connallon and Clark 2012; Williams and Day 2003; for antagonistic pleiotropy in the context of sexual antagonism under panmixia, see Zajitschek and Connallon 2017, 2018). Here, models have also highlighted the importance of component-specific dominance effects, such that antagonistic effects are recessive for each fitness component, in facilitating balancing selection (Rose 1982, 1985; Curtsinger et al. 1994; Connallon and Chenoweth 2019). But these models assume that populations are well mixed and that genotype frequencies prior to antagonistic selection are at Hardy-Weinberg equilibrium (Rose 1982, 1985; Curtsinger et al. 1994). From our results, we expect that limited dispersal will also reduce the scope for polymorphism from antagonistic pleiotropy and may therefore influence the evolution of a wide range of traits involved in fitness trade-offs (Roff 2002), such as viability and expression of secondary sexual characters (Zahavi 1975; Lande 1981; Kirkpatrick 1982; Grafen 1990; Iwasa et al. 1991; Iwasa and Pomiankowski 1994), resource acquisition (Van Noordwijk and de Jong 1986), and reproductive investment and life span (Williams 1957; Williams and Day 2003; Nussey et al. 2013).

Our model, which has allowed us to produce tractable and intuitive results about intralocus sexual conflict under limited dispersal, relies on several assumptions that are of course unrealistic for many natural populations. In particular, while our model allows us to vary demographic parameters such as adult sex ratio and dispersal regime (immigrant sex ratio and total dispersal rate), these quantities are not free to vary among groups or evolve. In natural populations, however, parameters such as sex ratio and sex-specific dispersal are correlated by ecological dynamics, and their interdependence will lead them to coevolve. Investigating how such eco-evo feedbacks between local demography and sexual antagonism shape mating system evolution would be an interesting avenue for future work (for general considerations, see Giery and Layman 2019; Svensson 2019; for such an eco-evo model of sexual antagonism under panmixia, see de Vries and Caswell 2019; for analyses of selection on traits influencing demography in subdivided populations, see Rousset and Ronce 2004; Mullon and Lehmann 2018). In this context, it would also be relevant to relax our assumption that female fecundity is large in order to consider the effects of sexspecific reproductive variance (Mullon et al. 2014), which is common in nature (Clutton-Brock 2007) and is especially important for selection in subdivided populations (Lehmann and Balloux 2007). Another assumption of our model is environmental homogeneity between patches (i.e., that selection on males and females is consistent across space). In contrast to our results, a previous model has shown that in the absence of genetic structure (i.e., assuming infinite patch size), sex-biased dispersal in spatially heterogeneous environments can lead sexually antagonistic selection to favor adaptation in the philopatric sex over the dispersing one (Connallon et al. 2019). This is because under spatial heterogeneity the dispersing sex experiences greater variance in selection pressure, weakening the overall strength of directional selection in this sex. To better understand the contrasting effects of spatial variation and genetic structure, a valuable extension of our model would therefore be to consider heterogeneous subdivided populations consisting of finite groups (although 
note that this is significantly more complicated mathematically; e.g., Rousset and Ronce 2004; Lehmann et al. 2016).

In conclusion, through its effects on inbreeding, kin competition, and genetic drift, limited dispersal qualitatively alters the segregation of sexually antagonistic alleles, narrowing the scope for sexually antagonistic polymorphism and changing the nature of intralocus conflict. Dispersal patterns, and in particular their sex specificity, are therefore a relevant consideration for the genetic and ecological conditions expected to lead to sexually antagonistic variation as well as for the nature of the alleles underlying such variation. More broadly, our results reinforce the general notion that spatial demography and population structure are important factors for population genetic dynamics and the maintenance of nonneutral variation (e.g., Wright 1949; Hamilton 1964; Rousset 2004; Hartl and Clark 2007; Charlesworth and Charlesworth 2010).

\section{Acknowledgments}

We thank Carl Mackintosh, Max Reuter, Stu Wigby, Henry Barton, Daniel Bolnick, Stephen Chenoweth, and two anonymous reviewers for comments and discussion on the manuscript; Ben Haller for advice on simulations; and the UK Natural Environment Research Council and the Swiss National Science Foundation (PCEFP3181243 to C.M.) for funding.

\section{Statement of Authorship}

E.O.F., V.S., and C.M. conceived the study. E.O.F. and C.M. performed the analyses. E.O.F. and C.M. wrote the manuscript with input from V.S.

\section{Data and Code Availability}

Code is available online in a zip file and on Zenodo (Flintham 2021).

\section{Literature Cited}

Albert, A. Y., and S. P. Otto. 2005. Sexual selection can resolve sexlinked sexual antagonism. Science 310:119-121.

Arnqvist, G. 2011. Assortative mating by fitness and sexually antagonistic genetic variation. Evolution 65:2111-2116.

Barson, N. J., T. Aykanat, K. Hindar, M. Baranski, G. H. Bolstad, P. Fiske, C. Jacq, et al. 2015. Sex-dependent dominance at a single locus maintains variation in age at maturity in salmon. $\mathrm{Na}$ ture 528:405-408.

Barton, N. H. 2001. The evolutionary consequences of gene flow and local adaptation: future approaches. Pages 329-340 in J. Clobert, E. Danchin, A. A. Dhondt, and J. D. Nichols, eds. Dispersal. Oxford University Press, New York.
Berry, O., M. D. Tocher, D. M. Gleeson, and S. D. Sarre. 2005. Effect of vegetation matrix on animal dispersal: genetic evidence from a study of endangered skinks. Conservation Biology 19:855-864.

Böhme, M. U., N. Schneeweiß, U. Fritz, M. Schlegel, and T. U. Berendonk. 2007. Small edge populations at risk: genetic diversity of the green lizard (Lacerta viridis viridis) in Germany and implications for conservation management. Conservation Genetics 8:555-563.

Bonduriansky, R., and S. F. Chenoweth. 2009. Intralocus sexual conflict. Trends in Ecology and Evolution 24:280-288.

Brommer, J. E., M. Kirkpatrick, A. Qvarnström, and L. Gustafsson. 2007. The intersexual genetic correlation for lifetime fitness in the wild and its implications for sexual selection. PLoS ONE 2:e744.

Caballero, A. 1994. Developments in the prediction of effective population size. Heredity 73:657-679.

Canestrelli, D., A. Verardi, and G. Nascetti. 2007. Genetic differentiation and history of populations of the Italian treefrog Hyla intermedia: lack of concordance between mitochondrial and nuclear markers. Genetica 130:241-255.

Cegelski, C., L. Waits, and N. Anderson. 2003. Assessing population structure and gene flow in Montana wolverines (Gulo gulo) using assignment-based approaches. Molecular Ecology 12:2907-2918.

Charlesworth, B., and D. Charlesworth. 2010. Elements of evolutionary genetics. Roberts, Greenwood Village, CO.

Charlesworth, B., J. A. Coyne, and N. H. Barton. 1987. The relative rates of evolution of sex chromosomes and autosomes. American Naturalist 130:113-146.

Chippindale, A. K., J. R. Gibson, and W. R. Rice. 2001. Negative genetic correlation for adult fitness between sexes reveals ontogenetic conflict in Drosophila. Proceedings of the National Academy of Sciences of the USA 98:1671-1675.

Clobert, J., E. Danchin, A. A. Dhondt, and J. D. Nichols, eds. 2001. Dispersal. Oxford University Press, New York.

Clutton-Brock, T. 2007. Sexual selection in males and females. Science 318:1882-1885.

Connallon, T., and S. F. Chenoweth. 2019. Dominance reversals and the maintenance of genetic variation for fitness. PLoS Biology 17:e3000118.

Connallon, T., and A. G. Clark. 2012. A general population genetic framework for antagonistic selection that accounts for demography and recurrent mutation. Genetics 190:1477-1489.

- 2014. Evolutionary inevitability of sexual antagonism. Proceedings of the Royal Society B 281:20132123.

Connallon, T., S. Sharma, and C. Olito. 2019. Evolutionary consequences of sex-specific selection in variable environments: four simple models reveal diverse evolutionary outcomes. American Naturalist 193:93-105.

Curtsinger, J. W., P. M. Service, and T. Prout. 1994. Antagonistic pleiotropy, reversal of dominance, and genetic polymorphism. American Naturalist 144:210-228.

Débarre, F., and S. Gandon. 2011. Evolution in heterogeneous environments: between soft and hard selection. American Naturalist 177:E84-E97.

de Vries, C., and H. Caswell. 2019. Selection in two-sex stagestructured populations: genetics, demography, and polymorphism. Theoretical Population Biology 130:160-169.

Dobson, F. S. 1982. Competition for mates and predominant juvenile male dispersal in mammals. Animal Behaviour 30:1183-1192.

Eyer, P.-A., A. J. Blumenfeld, and E. L. Vargo. 2019. Sexually antagonistic selection promotes genetic divergence between males 
and females in an ant. Proceedings of the National Academy of Sciences of the USA 116:24157-24163.

Flintham, E. 2021. eoflintham/Dispersal_alters_the_nature_and_scope _of_sexually_antagonistic_variation: code archiving AmNat 2021. Version v1.0.0. Zenodo, https://doi.org/10.5281/zenodo.4463460.

Foerster, K., T. Coulson, B. C. Sheldon, J. M. Pemberton, T. H. Clutton-Brock, and L. E. Kruuk. 2007. Sexually antagonistic genetic variation for fitness in red deer. Nature 447:1107-1110.

Frank, S. A. 1998. Foundations of social evolution. Monographs in Behavior and Ecology. Princeton University Press, Princeton, NJ.

Fry, J. D. 2010. The genomic location of sexually antagonistic variation: some cautionary comments. Evolution 64:1510-1516.

Gavrilets, S., and W. R. Rice. 2006. Genetic models of homosexuality: generating testable predictions. Proceedings of the Royal Society B 273:3031-3038.

Gibson, J. R., A. K. Chippindale, and W. R. Rice. 2002. The X chromosome is a hot spot for sexually antagonistic fitness variation. Proceedings of the Royal Society B 269:499-505.

Giery, S. T., and C. A. Layman. 2019. Ecological consequences of sexually selected traits: an eco-evolutionary perspective. Quarterly Review of Biology 94:29-74.

Grafen, A. 1990. Biological signals as handicaps. Journal of Theoretical Biology 144:517-546.

Greenwood, P. J. 1980. Mating systems, philopatry and dispersal in birds and mammals. Animal Behaviour 28:1140-1162.

Grieshop, K., and G. Arnqvist. 2018. Sex-specific dominance reversal of genetic variation for fitness. PLoS Biology 16:e2006810.

Hager, R., J. M. Cheverud, L. J. Leamy, and J. B. Wolf. 2008. Sex dependent imprinting effects on complex traits in mice. BMC Evolutionary Biology 8:303.

Haller, B. C., and P. W. Messer. 2019. SLiM 3: forward genetic simulations beyond the Wright-Fisher model. Molecular Biology and Evolution 36:632-637.

Hamilton, W. D. 1964. The genetical evolution of social behaviour. II. Journal of Theoretical Biology 7:17-52.

- 1967. Extraordinary sex ratios. Science 156:477-488.

Hammond, R. L., L. J. Lawson Handley, B. J. Winney, M. W Bruford, and N. Perrin. 2006. Genetic evidence for female-biased dispersal and gene flow in a polygynous primate. Proceedings of the Royal Society B 273:479-484.

Hansson, B., S. Bensch, and D. Hasselquist. 2003. A new approach to study dispersal: immigration of novel alleles reveals femalebiased dispersal in great reed warblers. Molecular Ecology 12:631637.

Harrison, X. A., J. E. York, and A. J. Young. 2014. Population genetic structure and direct observations reveal sex-reversed patterns of dispersal in a cooperative bird. Molecular Ecology 23:57405755.

Hartl, D. L., and A. G. Clark. 2007. Principles of population genetics. 4th ed. Vol. 116. Sinauer, Sunderland, MA.

Harts, A. M., L. E. Schwanz, and H. Kokko. 2014. Demography can favour female-advantageous alleles. Proceedings of the Royal Society B 281:20140005.

Hitchcock, T. J., and A. Gardner. 2020. A gene's-eye view of sexual antagonism. Proceedings of the Royal Society B 287:20201633.

Iwasa, Y., and A. Pomiankowski. 1994. The evolution of mate preferences for multiple sexual ornaments. Evolution 48:853-867.

Iwasa, Y., A. Pomiankowski, and S. Nee. 1991. The evolution of costly mate preferences. II. The "handicap" principle. Evolution 45:1431-1442.
Janicke, T., I. K. Häderer, M. J. Lajeunesse, and N. Anthes. 2016. Darwinian sex roles confirmed across the animal kingdom. Science Advances 2:e1500983.

Jaquiéry, J., C. Rispe, D. Roze, F. Legeai, G. Le Trionnaire, S. Stoeckel, L. Mieuzet, et al. 2013. Masculinization of the X chromosome in the pea aphid. PLoS Genetics 9:e1003690-15.

Jordan, C. Y., and D. Charlesworth. 2012. The potential for sexually antagonistic polymorphism in different genome regions. Evolution 66:505-516.

Jordan, C. Y. and T. Connallon. 2014. Sexually antagonistic polymorphism in simultaneous hermaphrodites. Evolution 68:3555-3569.

Kasimatis, K. R., P. L. Ralph, and P. C. Phillips. 2019. Limits to genomic divergence under sexually antagonistic selection. G3: Genes, Genomes, Genetics 9:3813-3824.

Kidwell, J., M. Clegg, F. Stewart, and T. Prout. 1977. Regions of stable equilibria for models of differential selection in the two sexes under random mating. Genetics 85:171-183.

Kirkpatrick, M. 1982. Sexual selection and the evolution of female choice. Evolution 36:1-12.

Kirkwood, T. B., and M. R. Rose. 1991. Evolution of senescence: late survival sacrificed for reproduction. Philosophical Transactions of the Royal Society B 332:15-24.

Lande, R. 1981. Models of speciation by sexual selection on polygenic traits. Proceedings of the National Academy of Sciences of the USA 78:3721-3725.

Lehmann, L., and F. Balloux. 2007. Natural selection on fecundity variance in subdivided populations: kin selection meets bet hedging. Genetics 176:361-377.

Lehmann, L., C. Mullon, E. Akçay, and J. Van Cleve. 2016. Invasion fitness, inclusive fitness, and reproductive numbers in heterogeneous populations. Evolution 70:1689-1702.

Lewis, Z., N. Wedell, and J. Hunt. 2011. Evidence for strong intralocus sexual conflict in the Indian meal moth, Plodia interpunctella. Evolution 65:2085-2097.

Li, X.-Y., and L. Holman. 2018. Evolution of female choice under intralocus sexual conflict and genotype-by-environment interactions. Philosophical Transactions of the Royal Society B 373: 20170425.

Li, X.-Y., and H. Kokko. 2019. Sex-biased dispersal: a review of the theory. Biological Reviews 94:721-736.

Lucotte, E. A., R. Laurent, E. Heyer, L. Segurel, and B. Toupance. 2016. Detection of allelic frequency differences between the sexes in humans: a signature of sexually antagonistic selection. Genome Biology and Evolution 8:1489-1500.

Mainguy, J., S. D. Coté, M. Festa-Bianchet, and D. W. Coltman. 2009. Father-offspring phenotypic correlations suggest intralocus sexual conflict for a fitness-linked trait in a wild sexually dimorphic mammal. Proceedings of the Royal Society B 276: 4067-4075.

Mullon, C., and L. Lehmann. 2018. Eco-evolutionary dynamics in metacommunities: ecological inheritance, helping within species, and harming between species. American Naturalist 192:664-686.

Mullon, C., A. Pomiankowski, and M. Reuter. 2012. The effects of selection and genetic drift on the genomic distribution of sexually antagonistic alleles. Evolution 66:3743-3753.

Mullon, C., M. Reuter, and L. Lehmann. 2014. The evolution and consequences of sex-specific reproductive variance. Genetics 196:235-252.

Nussey, D. H., H. Froy, J.-F. Lemaitre, J.-M. Gaillard, and S. N. Austad. 2013. Senescence in natural populations of animals: 
widespread evidence and its implications for bio-gerontology. Ageing Research Reviews 12:214-225.

Otto, S. P., J. R. Pannell, C. L. Peichel, T.-L. Ashman, D. Charlesworth, A. K. Chippindale, L. F. Delph, R. F. Guerrero, S. V. Scarpino, and B. F. McAllister. 2011. About par: the distinct evolutionary dynamics of the pseudoautosomal region. Trends in Genetics 27:358-367.

Owen, A. 1953. A genetical system admitting of two distinct stable equilibria under natural selection. Heredity 7:97-102.

Parker, G. A. 1979. Sexual selection and sexual conflict. Pages 123166 in M. S. Blum and N. A. Blum. Sexual selection and reproductive competition in insects. Elsevier, Amsterdam.

Pearse, D. E., N. J. Barson, T. Nome, G. Gao, M. A. Campbell, A. Abadía-Cardoso, E. C. Anderson, et al. 2019. Sex-dependent dominance maintains migration supergene in rainbow trout. Nature Ecology and Evolution 3:1731-1742.

Perrin, N., and V. Mazalov. 2000. Local competition, inbreeding, and the evolution of sex-biased dispersal. American Naturalist 155:116-127.

Poissant, J., A. J. Wilson, and D. W. Coltman. 2010. Sex-specific genetic variance and the evolution of sexual dimorphism: a systematic review of cross-sex genetic correlations. Evolution 64:97-107.

Ramachandran, S., N. A. Rosenberg, M. W. Feldman, and J. Wakeley. 2008. Population differentiation and migration: coalescence times in a two-sex island model for autosomal and X-linked loci. Theoretical Population Biology 74:291-301.

Rice, W. R. 1984. Sex chromosomes and the evolution of sexual dimorphism. Evolution 38:735-742.

Roff, D. A. 2002. Life history evolution. Sinauer, Sunderland, MA.

Rose, M. R. 1982. Antagonistic pleiotropy, dominance, and genetic variation. Heredity 48:63-78.

1985. Life history evolution with antagonistic pleiotropy and overlapping generations. Theoretical Population Biology 28:342-358.

Rousset, F. 2004. Genetic structure and selection in subdivided populations. Princeton University Press, Princeton, NJ.

Rousset, F., and O. Ronce. 2004. Inclusive fitness for traits affecting metapopulation demography. Theoretical Population Biology 65:127-141.

Roze, D., and F. Rousset. 2003. Selection and drift in subdivided populations: a straightforward method for deriving diffusion approximations and applications involving dominance, selfing and local extinctions. Genetics 165:2153-2166.

Ruzicka, F., and T. Connallon. 2020. Is the X chromosome a hot spot for sexually antagonistic polymorphisms? biases in current empirical tests of classical theory. Proceedings of the Royal Society B 287:20201869.

Ruzicka, F., M. S. Hill, T. M. Pennell, I. Flis, F. C. Ingleby, R. Mott, K. Fowler, E. H. Morrow, and M. Reuter. 2019. Genome-wide sexually antagonistic variants reveal long-standing constraints on sexual dimorphism in fruit flies. PLoS Biology 17:e3000244.

Schulte-Hostedde, A. I., H. L. Gibbs, and J. S. Millar. 2001. Microgeographic genetic structure in the yellow-pine chipmunk (Tamias amoenus). Molecular Ecology 10:1625-1631.

Schulte-Hostedde, A. I., J. S. Millar, and H. L. Gibbs. 2002. Femalebiased sexual size dimorphism in the yellow-pine chipmunk (Tamias amoenus): sex-specific patterns of annual reproductive success and survival. Evolution 56:2519-2529.

Shields, W. M. 1987. Dispersal and mating systems: investigating their causal connections. Pages 3-24 in D. B. Chepko-Sade and
Z. T. Halpin. Mammalian dispersal patterns: the effects of social structure on population genetics. University of Chicago Press, Chicago.

Singh, A., and D. Punzalan. 2018. The strength of sex-specific selection in the wild. Evolution 72:2818-2824.

Spencer, H. G., and N. K. Priest. 2016. The evolution of sex-specific dominance in response to sexually antagonistic selection. American Naturalist 187:658-666.

Svensson, E. I. 2019. Eco-evolutionary dynamics of sexual selection and sexual conflict. Functional Ecology 33:60-72.

Svensson, E. I., A. G. McAdam, and B. Sinervo. 2009. Intralocus sexual conflict over immune defense, gender load, and sex-specific signaling in a natural lizard population. Evolution 63:3124-3135.

Tarka, M., M. Åkesson, D. Hasselquist, and B. Hansson. 2014. Intralocus sexual conflict over wing length in a wild migratory bird. American Naturalist 183:62-73.

Taylor, P. D., and A. J. Irwin. 2000. Overlapping generations can promote altruistic behavior. Evolution 54:1135-1141.

Tazzyman, S. J., and J. K. Abbott. 2015. Self-fertilization and inbreeding limit the scope for sexually antagonistic polymorphism. Journal of Evolutionary Biology 28:723-729.

Trochet, A., E. A. Courtois, V. M. Stevens, M. Baguette, A. Chaine, D. S. Schmeller, J. Clobert, and J. J. Wiens. 2016. Evolution of sexbiased dispersal. Quarterly Review of Biology 91:297-320.

Vähä, J.-P., J. Erkinaro, E. Niemelä, and C. R. Primmer. 2007. Lifehistory and habitat features influence the within-river genetic structure of Atlantic salmon. Molecular Ecology 16:2638-2654.

- 2008. Temporally stable genetic structure and low migration in an Atlantic salmon population complex: implications for conservation and management. Evolutionary Applications 1:137154.

Van Cleve, J., M. W. Feldman, and L. Lehmann. 2010. How demography, life history, and kinship shape the evolution of genomic imprinting. American Naturalist 176:440-455.

Van Noordwijk, A. J., and G. de Jong. 1986. Acquisition and allocation of resources: their influence on variation in life history tactics. American Naturalist 128:137-142.

Vicoso, B., and B. Charlesworth. 2009. Effective population size and the faster-X effect: an extended model. Evolution 63:2413-2426.

Wang, J. 1997. Effective size and F-statistics of subdivided populations. II. Dioecious species. Genetics 146:1465-1474.

Wang, J., and A. Caballero. 1999. Developments in predicting the effective size of subdivided populations. Heredity 82:212-226.

Wang, W., J. Li, H. Wang, Y. Ran, and H. Wu. 2020. Genetic evidence reveals male-biased dispersal of the Omei tree frog. Journal of Zoology 310:201-209.

Wellband, K., C. Mérot, T. Linnansaari, J. Elliott, R. A. Curry, and L. Bernatchez. 2019. Chromosomal fusion and life historyassociated genomic variation contribute to within-river local adaptation of Atlantic salmon. Molecular Ecology 28:1439-1459.

Williams, G. C. 1957. Pleiotropy, natural selection, and the evolution of senescence. Evolution 11:398-411.

Williams, P. D., and T. Day. 2003. Antagonistic pleiotropy, mortality source interactions, and the evolutionary theory of senescence. Evolution 57:1478-1488.

Woxvold, I. A., G. J. Adcock, and R. A. Mulder. 2006. Fine-scale genetic structure and dispersal in cooperatively breeding apostlebirds. Molecular Ecology 15:3139-3146.

Wright, S. 1922. Coefficients of inbreeding and relationship. American Naturalist 56:330-338. 
16:97-159.

1949. The genetical structure of populations. Annals of Eugenics 15:323-354.

Zahavi, A. 1975. Mate selection - a selection for a handicap. Journal of Theoretical Biology 53:205-214.

Zajitschek, F., and T. Connallon. 2017. Partitioning of resources: the evolutionary genetics of sexual conflict over resource acqui- sition and allocation. Journal of Evolutionary Biology 30:826838.

2018. Antagonistic pleiotropy in species with separate sexes, and the maintenance of genetic variation in life-history traits and fitness. Evolution 72:1306-1316.

Associate Editor: Stephen F. Chenoweth Editor: Daniel I. Bolnick

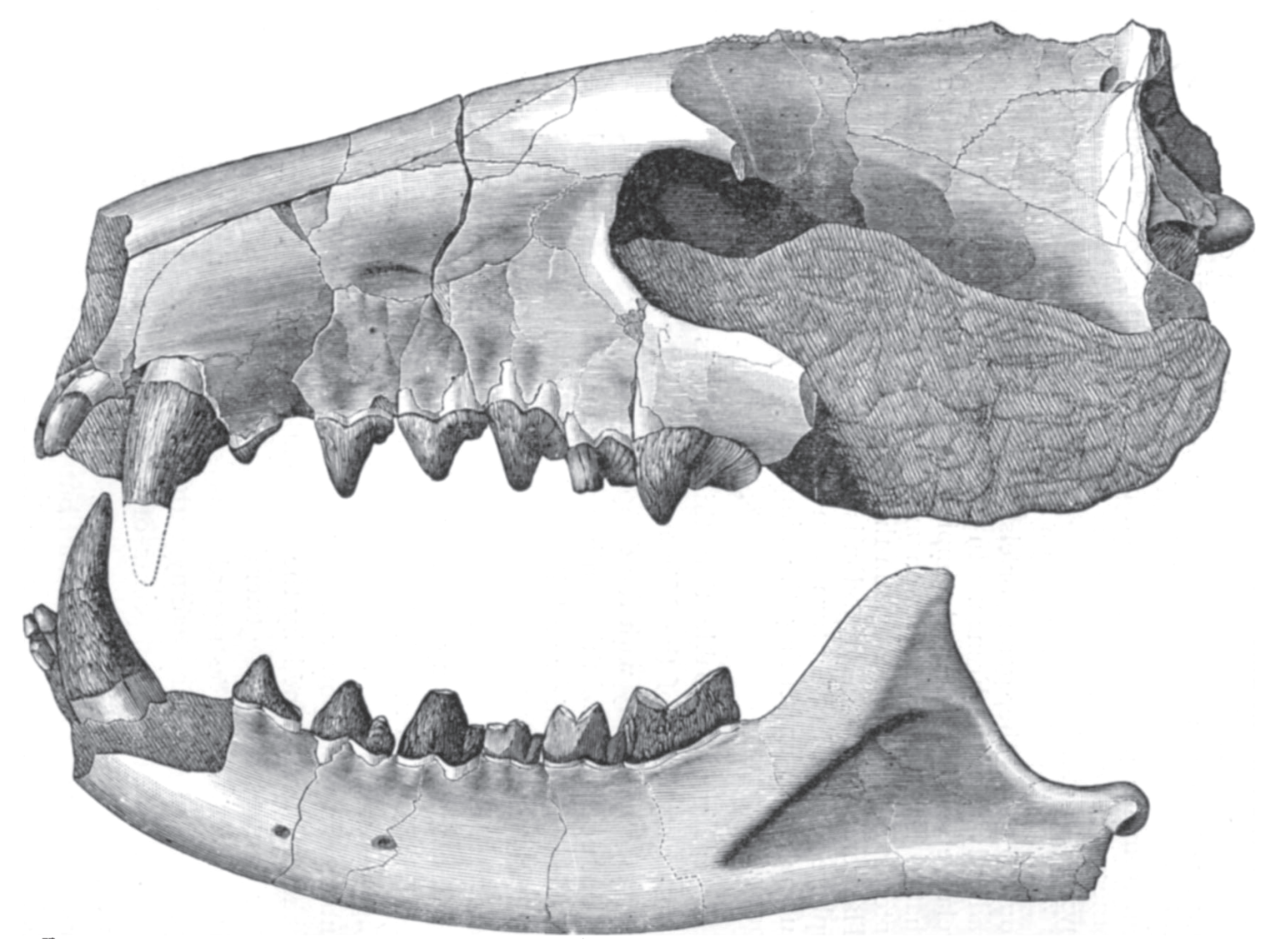

"But one genus of this family has been thus far described, the Hyænodon of Laizer and Parieu. . . The species range in size from that of the H. vulpinus Gerv., which equals a red fox, to that of an American black bear, as the H. herberti Filhol, and H. horridus Leidy [figured]. The latter species is from the bad lands of Nebraska." From "The Creodonta" by E. D. Cope (The American Naturalist, 1884, 18:344-353). 


\title{
Appendices A \& B from "Dispersal alters the nature and scope of sexually antagonistic variation" (The American Naturalist).
}

\author{
Ewan O Flintham ${ }^{1}$, Vincent Savolainen ${ }^{1, *}$, and Charles Mullon ${ }^{2}$ \\ ${ }^{1}$ Department of Life Sciences, Silwood Park Campus, Imperial College London, Ascot, SL5 \\ 7PY United Kingdom \\ ${ }^{2}$ Department of Ecology and Evolution, University of Lausanne, 1015 Lausanne, Switzerland \\ Correspondence: v.savolainen@imperial.ac.uk
}


Flintham et al., "Dispersal and sexual antagonism"

\section{Appendix}

\section{A Allele frequency change in a subdivided dioecious population}

In this appendix, we derive the change in frequency over one generation for an allele at an autosomal and sex-linked locus, assuming that selection is weak. Our derivation follows closely previous analyses of population genetics models in subidvided populations (Roze and Rousset, 2003; Rousset, 2004, in particular Van Cleve et al., 2010).

\section{A.1 Model}

We first provide further details on our modelling assumptions, which are necessary for our derivations. Recall we consider a dioecious population that is divided among a large number $n_{\mathrm{g}}$ of groups, each composed of $n_{\mathrm{m}}$ and $n_{\mathrm{f}}$ adult males and females, respectively. The life-cycle of this population is as follows. 1) Adults mate randomly within their group, females then produce a large number $k$ of male and female juveniles in equal proportions (i.e. unbiased sex ratio at birth). 2) Independently of one another, each juvenile either remains in its natal group, or disperses to another randomly chosen group. 3) Adults die, and male and female juveniles compete to fill the $n_{\mathrm{m}}$ and $n_{\mathrm{f}}$ open breeding spots in each group (so competition occurs within the sexes and within groups).

Each individual carries a quantitative phenotype that is genetically-encoded and that determines the competitiveness of a juvenile to settle in a group (step 3 of the life-cycle). We will separately consider evolution at two loci that influence this phenotype: at an autosomal and at a sex-linked locus (on the $\mathrm{X}$-chromosome in an $\mathrm{XY}$ species or the $\mathrm{Z}$ chromosome in a $\mathrm{ZW}$ species). In either case, two alleles $A$ and $a$ with sexually antagonistic effects segregate in the population. We are interested in deriving the change in frequency in the whole population of the allele $A$. For mathematical convenience, the population is censused in juveniles before dispersal (between steps 1 and 2 of the life-cycle).

\section{A.2 Autosomal locus}

\section{A.2.1 Genotypes}

To denote the genotype of males and females, we let $p_{i j 1}^{u} \in\{0,1\}$ and $p_{i j 2}^{u} \in\{0,1\}$ be the frequency of $A$ at the maternally and paternally inherited locus, respectively, of juvenile $j \in\left\{1, \ldots, n_{\mathrm{f}} k / 2\right\}$ of sex $u \in\{\mathrm{m}, \mathrm{f}\}$ residing in group $i \in\left\{1, \ldots, n_{\mathrm{g}}\right\}$ (recall that $k$ is the total fecundity of a female, so that within a group there are $n_{\mathrm{f}} k / 2$ male and $n_{\mathrm{f}} k / 2$ female juveniles after reproduction and before dispersal). The frequency of $A$ in such an individual then is

$$
p_{i j}^{u}=\frac{p_{i j 1}^{u}+p_{i j 2}^{u}}{2} \in\{0,1 / 2,1\} \text {. }
$$

For example, if male 2 in group 1 has inherited $A$ from its mother and $a$ from its father, then $p_{121}^{\mathrm{m}}=1$, $p_{122}^{\mathrm{m}}=0$, and $p_{12}^{\mathrm{m}}=1 / 2$. The average frequency of $A$ in individuals of sex $u$ can then be defined as

$$
p^{u}=\overline{p_{i j}^{u}},
$$

where the upper bar means the average over all juveniles $j$ and over all groups $i$ throughout. 
Flintham et al., "Dispersal and sexual antagonism"

\begin{tabular}{lccc}
\hline & $a a$ & $A a$ & $A A$ \\
\hline male phenotype & 1 & $1-\delta h_{\mathrm{m}} c_{\mathrm{m}}$ & $1-\delta c_{\mathrm{m}}$ \\
female phenotype & $1-\delta c_{\mathrm{f}}$ & $1-\delta h_{\mathrm{f}} c_{\mathrm{f}}$ & 1 \\
\hline
\end{tabular}

Table A-1: Male and female phenotypes according to genotype.

\section{A.2.2 Phenotypes}

The evolving phenotype determines the competitiveness of male and female juveniles. We assume that baseline competitiveness is 1 and that allele $A$ is detrimental to males (causing a decrease in competitiveness $\delta c_{\mathrm{m}}$ in homozygotes and $\delta h_{\mathrm{m}} c_{\mathrm{m}}$ in heterozygotes, Table A-1). Conversely, allele $a$ is detrimental to females causing a decrease in competitiveness $\delta c_{\mathrm{f}}$ in homozygotes and $\delta h_{\mathrm{f}} c_{\mathrm{f}}$ in heterozygotes, Table A-1). Parameters $c_{\mathrm{m}}>0$ and $c_{\mathrm{f}}>0$ respectively capture the cost of the detrimental allele in male and female homozygotes ( $\delta>0$ tunes the strength of selection), while parameters $h_{\mathrm{m}}$ and $h_{\mathrm{f}}$ determine the dominance or penetrance of the detrimental allele in males and females, respectively. We can then write the phenotype $z_{i j}^{u}$ of juvenile $j$ of $\operatorname{sex} u$ that resides in group $i$ in terms of the frequency of allele $A$ in this individual as

$$
\begin{aligned}
& z_{i j}^{\mathrm{m}}=1-\delta c_{\mathrm{m}}\left(2 h_{\mathrm{m}} p_{i j}^{\mathrm{m}}+\left(1-2 h_{\mathrm{m}}\right) p_{i j 1}^{\mathrm{m}} p_{i j 2}^{\mathrm{m}}\right) \\
& z_{i j}^{\mathrm{f}}=1+\delta c_{\mathrm{f}}\left(-1+2\left(1-h_{\mathrm{f}}\right) p_{i j}^{\mathrm{f}}+\left(1-2\left(1-h_{\mathrm{f}}\right)\right) p_{i j 1}^{\mathrm{f}} p_{i j 2}^{\mathrm{f}}\right),
\end{aligned}
$$

according to Table A-1.

\section{A.2.3 Weighted allele frequency change}

To track the dynamics of the frequencies of the $A$ allele in the population, we consider the dynamics of a reproductive-value weighted average $p$ of male and female frequencies,

$$
p=\alpha p^{\mathrm{f}}+(1-\alpha) p^{\mathrm{m}}
$$

where $\alpha$ and $1-\alpha$ are the female and male reproductive values, respectively (e.g. Roze and Rousset, 2003). This weighting ensures that in the absence of selection (when $\delta=0$ ), the change in $p$ is zero. For an autosomal locus, males and females have equal reproductive values: $\alpha=1 / 2$.

Following Roze and Rousset (2003) (adapting their eq. 35 for an autosomal locus) and Van Cleve et al. (2010) (their eq. 2), the change over one iteration of the life-cycle in weighted allele frequency change is

$\Delta p=\frac{1}{n_{\mathrm{g}}} \sum_{i=1}^{n_{\mathrm{g}}} \frac{1}{n_{\mathrm{f}} k / 2}\left[\alpha\left(\sum_{j=1}^{n_{\mathrm{f}} k / 2} w_{i j}^{\mathrm{f} \rightarrow \mathrm{f}} \frac{p_{i j}^{\mathrm{f}}}{2}+\sum_{j=1}^{n_{\mathrm{f}} k / 2} w_{i j}^{\mathrm{m} \rightarrow \mathrm{f}} \frac{p_{i j}^{\mathrm{m}}}{2}\right)+(1-\alpha)\left(\sum_{j=1}^{n_{\mathrm{f}} k / 2} w_{i j}^{\mathrm{f} \rightarrow \mathrm{m}} \frac{p_{i j}^{\mathrm{f}}}{2}+\sum_{j=1}^{n_{\mathrm{f}} k / 2} w_{i j}^{\mathrm{m} \rightarrow \mathrm{m}} \frac{p_{i j}^{\mathrm{m}}}{2}\right)\right]-p$,

where $w_{i j}^{u \rightarrow v}$ is the expected number of juveniles of sex $v$ produced by juvenile $j$ of sex $u$ in group $i$ (before dispersal) and the division of individual gene frequencies by 2 reflects that a gene has a probability of $1 / 2$ to be passed to an offspring at an autosomal locus (with no segregation bias). There are thus four relevant fitness functions: $w_{i j}^{\mathrm{f} \rightarrow \mathrm{f}}, w_{i j}^{\mathrm{m} \rightarrow \mathrm{f}}, w_{i j}^{\mathrm{f} \rightarrow \mathrm{m}}$, and $w_{i j}^{\mathrm{m} \rightarrow \mathrm{m}}$. We consider these fitness functions in more detail later (section A.2.5). But first note that when sex ratio at birth is equal, a 
juvenile of either sex is expected to produce the same numbers of male and female juvenile so that $w_{i j}^{\mathrm{f} \rightarrow \mathrm{f}}=w_{i j}^{\mathrm{f} \rightarrow \mathrm{m}}=w_{i j}^{\mathrm{f}} / 2$ and $w_{i j}^{\mathrm{m} \rightarrow \mathrm{f}}=w_{i j}^{\mathrm{m} \rightarrow \mathrm{m}}=w_{i j}^{\mathrm{m}} / 2$, where $w_{i j}^{\mathrm{f}}$ and $w_{i j}^{\mathrm{m}}$ are the expected total numbers of juveniles produces by a female and male juveniles, respectively. Substituting for these total fitness expressions and for reproductive value $\alpha=1 / 2$ into eq. (A-5), we obtain

$$
\Delta p=\frac{1}{4 n_{\mathrm{g}}} \sum_{i=1}^{n_{\mathrm{g}}} \frac{1}{n_{\mathrm{f}} k / 2}\left[\sum_{j=1}^{n_{\mathrm{f}} k / 2} w_{i j}^{\mathrm{f}} p_{i j}^{\mathrm{f}}+\sum_{j=1}^{n_{\mathrm{f}} k / 2} w_{i j}^{\mathrm{m}} p_{i j}^{\mathrm{m}}\right]-p
$$

for the change in weighted allele frequency.

\section{A.2.4 Selection gradient}

Next, we connect individual fitness with individual gene frequencies. To do so, first note that since competition is within groups and between individuals of the same sex, male and female fitness functions can in general be written as functions of three relevant phenotypic values,

$$
w_{i j}^{u}\left(z_{i j}^{u}, \bar{z}_{i 0}^{u}, \bar{z}^{u}\right)
$$

where $z_{i j}^{u}$ is the phenotype of the focal individual whose fitness is being considered (indexed $j$ of sex $u$ in group $i$ ); $\bar{z}_{i 0}^{u}$ is the average phenotype among the other individuals of the same sex born in group $i$ (i.e. excluding the focal individual); and $\bar{z}^{u}$ is the average phenotype of individuals of sex $u$ in the population (see eq. A-30 for an example of such fitness function). Second, we use the chain rule on these fitness functions to obtain,

$$
\begin{aligned}
& w_{i j}^{\mathrm{f}}=2+\delta\left(\frac{\partial w_{i j}^{\mathrm{f}}}{\partial z_{i j}^{\mathrm{f}}} \frac{\mathrm{d} z_{i j}^{\mathrm{f}}}{\mathrm{d} \delta}+\frac{\partial w_{i j}^{\mathrm{f}}}{\partial \bar{z}_{i 0}^{\mathrm{f}}} \frac{\mathrm{d} \bar{z}_{i 0}^{\mathrm{f}}}{\mathrm{d} \delta}+\frac{\partial w_{i j}^{\mathrm{f}}}{\partial \bar{z}^{\mathrm{f}}} \frac{\mathrm{d} \bar{z}^{\mathrm{f}}}{\mathrm{d} \delta}\right)+\mathscr{O}\left(\delta^{2}\right), \\
& w_{i j}^{\mathrm{m}}=2+\delta\left(\frac{\partial w_{i j}^{\mathrm{m}}}{\partial z_{i j}^{\mathrm{m}}} \frac{\mathrm{d} z_{i j}^{\mathrm{m}}}{\mathrm{d} \delta}+\frac{\partial w_{i j}^{\mathrm{m}}}{\partial \bar{z}_{i 0}^{\mathrm{m}}} \frac{\mathrm{d} \bar{z}_{i 0}^{\mathrm{m}}}{\mathrm{d} \delta}+\frac{\partial w_{i j}^{\mathrm{m}}}{\partial \bar{z}^{\mathrm{m}}} \frac{\mathrm{d} \bar{z}^{\mathrm{m}}}{\mathrm{d} \delta}\right)+\mathscr{O}\left(\delta^{2}\right)
\end{aligned}
$$

where 2 is the expected total number of juveniles produced by an individual under neutrality (i.e. one male and one female juvenile when $\delta=0$ ) and all derivatives are evaluated at $\delta=0$ (so at $z_{i j}^{\mathrm{f}}=\bar{z}_{i 0}^{\mathrm{f}}=$ $\bar{z}^{\mathrm{f}}=z_{i j}^{\mathrm{m}}=\bar{z}_{i 0}^{\mathrm{m}}=\bar{z}^{\mathrm{m}}=1$, from eq. A-3). The above equation may be further simplified by using the following relationships

$$
\begin{aligned}
& \frac{\partial w_{i j}^{\mathrm{f}}}{\partial z_{i j}^{\mathrm{f}}}+\frac{\partial w_{i j}^{\mathrm{f}}}{\partial \bar{z}_{i 0}^{\mathrm{f}}}+\frac{\partial w_{i j}^{\mathrm{f}}}{\partial \bar{z}^{\mathrm{f}}}=0 \\
& \frac{\partial w_{i j}^{\mathrm{m}}}{\partial z_{i j}^{\mathrm{m}}}+\frac{\partial w_{i j}^{\mathrm{m}}}{\partial \bar{z}_{i 0}^{\mathrm{m}}}+\frac{\partial w_{i j}^{\mathrm{m}}}{\partial \bar{z}^{\mathrm{m}}}=0,
\end{aligned}
$$

which hold because the population of each sex is constant, so that any fitness gain made by a focal individual due to a change of phenotype must be compensated by a decrease in fitness by the rest of the population (Rousset, 2004, p. 96).

Substituting eq. (A-9) into eq. (A-8), we obtain

$$
\begin{aligned}
& w_{i j}^{\mathrm{f}}=2+\delta \frac{\partial w_{i j}^{\mathrm{f}}}{\partial z_{i j}^{\mathrm{f}}}\left(\frac{\mathrm{d} z_{i j}^{\mathrm{f}}}{\mathrm{d} \delta}-\frac{\mathrm{d} \bar{z}^{\mathrm{f}}}{\mathrm{d} \delta}\right)+\delta \frac{\partial w_{i j}^{\mathrm{f}}}{\partial \bar{z}_{i 0}^{\mathrm{f}}}\left(\frac{\mathrm{d} \bar{z}_{i 0}^{\mathrm{f}}}{\mathrm{d} \delta}-\frac{\mathrm{d} \bar{z}^{\mathrm{f}}}{\mathrm{d} \delta}\right)+\mathscr{O}\left(\delta^{2}\right), \\
& w_{i j}^{\mathrm{m}}=2+\delta \frac{\partial w_{i j}^{\mathrm{m}}}{\partial z_{i j}^{\mathrm{m}}}\left(\frac{\mathrm{d} z_{i j}^{\mathrm{m}}}{\mathrm{d} \delta}-\frac{\mathrm{d} \bar{z}^{\mathrm{m}}}{\mathrm{d} \delta}\right)+\delta \frac{\partial w_{i j}^{\mathrm{m}}}{\partial \bar{z}_{i 0}^{\mathrm{m}}}\left(\frac{\mathrm{d} \bar{z}_{i 0}^{\mathrm{m}}}{\mathrm{d} \delta}-\frac{\mathrm{d} \bar{z}^{\mathrm{m}}}{\mathrm{d} \delta}\right)+\mathscr{O}\left(\delta^{2}\right) .
\end{aligned}
$$


Flintham et al., "Dispersal and sexual antagonism"

In turn, plugging eq. (A-10) into eq. (A-6), we obtain that the allele frequency change is

$$
\begin{aligned}
& \Delta p=\frac{1}{4} \delta {\left[\frac{\partial w_{i j}^{\mathrm{f}}}{\partial z_{i j}^{\mathrm{f}}} \overline{\left(\frac{\mathrm{d} z_{i j}^{\mathrm{f}}}{\mathrm{d} \delta}-\frac{\mathrm{d} \bar{z}^{\mathrm{f}}}{\mathrm{d} \delta}\right) p_{i j}^{\mathrm{f}}}+\frac{\partial w_{i j}^{\mathrm{f}}}{\partial \bar{z}_{i 0}^{\mathrm{f}}} \overline{\left(\frac{\mathrm{d} \bar{z}_{i 0}^{\mathrm{f}}}{\mathrm{d} \delta}-\frac{\mathrm{d} \bar{z}^{\mathrm{f}}}{\mathrm{d} \delta}\right) p_{i j}^{\mathrm{f}}}\right.} \\
&\left.+\frac{\partial w_{i j}^{\mathrm{m}}}{\partial z_{i j}^{\mathrm{m}}} \overline{\left(\frac{\mathrm{d} z_{i j}^{\mathrm{m}}}{\mathrm{d} \delta}-\frac{\mathrm{d} \bar{z}^{\mathrm{m}}}{\mathrm{d} \delta}\right) p_{i j}^{\mathrm{m}}}+\frac{\partial w_{i j}^{\mathrm{m}}}{\partial \bar{z}_{i 0}^{\mathrm{m}}} \overline{\left(\frac{\mathrm{d} \bar{z}_{i 0}^{\mathrm{m}}}{\mathrm{d} \delta}-\frac{\mathrm{d} \bar{z}^{\mathrm{m}}}{\mathrm{d} \delta}\right) p_{i j}^{\mathrm{m}}}\right]+\mathscr{O}\left(\delta^{2}\right),
\end{aligned}
$$

where the upper bar means the average over all juveniles and over all groups. From eq. (A-3), the averages in eq. (A-11) can be expressed in terms of products of allele frequencies within and between individuals:

$$
\begin{aligned}
& \overline{\left(\frac{\mathrm{d} z_{i j}^{\mathrm{f}}}{\mathrm{d} \delta}-\frac{\mathrm{d} \bar{z}^{\mathrm{f}}}{\mathrm{d} \delta}\right) p_{i j}^{\mathrm{f}}}=c_{\mathrm{f}}\left[2\left(1-h_{\mathrm{f}}\right)\left(\overline{\left(p_{i j}^{\mathrm{f}}\right)^{2}}-\left(p^{\mathrm{f}}\right)^{2}\right)+\left(1-2\left(1-h_{\mathrm{f}}\right)\right)\left(\overline{p_{i j 1}^{\mathrm{f}} p_{i j 2}^{\mathrm{f}} p_{i j}^{\mathrm{f}}}-\overline{p_{i j 1}^{\mathrm{f}} p_{i j 2}^{\mathrm{f}}} p^{\mathrm{f}}\right)\right] \\
& \overline{\left(\frac{\mathrm{d} \bar{z}_{i 0}^{\mathrm{f}}}{\mathrm{d} \delta}-\frac{\mathrm{d} \bar{z}^{\mathrm{f}}}{\mathrm{d} \delta}\right) p_{i j}^{\mathrm{f}}}=c_{\mathrm{f}}\left[2\left(1-h_{\mathrm{f}}\right)\left(\overline{p_{i 0}^{\mathrm{f}} p_{i j}^{\mathrm{f}}}-\left(p^{\mathrm{f}}\right)^{2}\right)+\left(1-2\left(1-h_{\mathrm{f}}\right)\right)\left(\overline{p_{i 01}^{\mathrm{f}} p_{i 02}^{\mathrm{f}} p_{i j}^{\mathrm{f}}}-\overline{p_{i j 1}^{\mathrm{f}} p_{i j 2}^{\mathrm{f}}} p^{\mathrm{f}}\right)\right],
\end{aligned}
$$

in females, where $p_{i 01}^{\mathrm{f}}$ (and $p_{i 02}^{\mathrm{f}}$ ) is the average frequency of the $A$ allele at the maternal (and paternal) locus of the neighbouring females of a focal female indexed $j$ in group $i$, and $p_{i 0}^{\mathrm{f}}=\left(p_{i 01}^{\mathrm{f}}+p_{i 02}^{\mathrm{f}}\right) / 2$. Similarly,

$$
\begin{aligned}
& \overline{\left(\frac{\mathrm{d} z_{i j}^{\mathrm{m}}}{\mathrm{d} \delta}-\frac{\mathrm{d} \bar{z}^{\mathrm{m}}}{\mathrm{d} \delta}\right) p_{i j}^{\mathrm{m}}}=-c_{\mathrm{m}}\left[2 h_{\mathrm{m}}\left(\overline{\left(p_{i j}^{\mathrm{m}}\right)^{2}}-\left(p^{\mathrm{m}}\right)^{2}\right)+\left(1-2 h_{\mathrm{m}}\right)\left(\overline{p_{i j 1}^{\mathrm{m}} p_{i j 2}^{\mathrm{m}} p_{i j}^{\mathrm{m}}}-\overline{p_{i j 1}^{\mathrm{m}} p_{i j 2}^{\mathrm{m}}} p^{\mathrm{m}}\right)\right] \\
& \overline{\left(\frac{\mathrm{d} \bar{z}_{i 0}^{\mathrm{m}}}{\mathrm{d} \delta}-\frac{\mathrm{d} \bar{z}^{\mathrm{m}}}{\mathrm{d} \delta}\right) p_{i j}^{\mathrm{m}}}=-c_{\mathrm{m}}\left[2 h_{\mathrm{m}}\left(\overline{p_{i 0}^{\mathrm{m}} p_{i j}^{\mathrm{m}}}-\left(p^{\mathrm{m}}\right)^{2}\right)+\left(1-2 h_{\mathrm{m}}\right)\left(\overline{p_{i 01}^{\mathrm{m}} p_{i 02}^{\mathrm{m}} p_{i j}^{\mathrm{m}}}-\overline{p_{i j 1}^{\mathrm{m}} p_{i j 2}^{\mathrm{m}}} p^{\mathrm{m}}\right)\right],
\end{aligned}
$$

in males, where $p_{i 01}^{\mathrm{m}}$ (and $p_{i 02}^{\mathrm{m}}$ ) is the average frequency of the $A$ allele at the maternal (and paternal) locus of the neighbouring males of a focal male indexed $j$ in group $i$, and $p_{i 0}^{\mathrm{m}}=\left(p_{i 01}^{\mathrm{m}}+p_{i 02}^{\mathrm{m}}\right) / 2$. We first simplify eqs. (A-12) and (A-14). Using the properties of indicator variables (that $\left(p_{i j 1}^{u}\right)^{2}=p_{i j 1}^{u}$ and $\left.\left(p_{i j 2}^{u}\right)^{2}=p_{i j 2}^{u}\right)$, we have

$$
\left(p_{i j}^{u}\right)^{2}=\frac{1}{2} p_{i j}^{u}+\frac{1}{2} p_{i j 1}^{u} p_{i j 2}^{u}
$$

so that

$$
\overline{\left(p_{i j}^{u}\right)^{2}}=\frac{1}{2} p^{u}+\frac{1}{2} \overline{p_{i j 1}^{u} p_{i j 2}^{u}} \text {. }
$$

Similarly, we have that

$$
\overline{p_{i j 1}^{u} p_{i j 2}^{u} p_{i j}^{u}}=\overline{p_{i j 1}^{u} p_{i j 2}^{u}} .
$$

Substituting eqs. (A-17) and (A-18) into eqs. (A-12) and (A-14), these read as

$$
\frac{\overline{\left(\frac{\mathrm{d} z_{i j}^{\mathrm{f}}}{\mathrm{d} \delta}-\frac{\mathrm{d} \bar{z}^{\mathrm{f}}}{\mathrm{d} \delta}\right) p_{i j}^{\mathrm{f}}}=c_{\mathrm{f}}\left[\left(1-p^{\mathrm{f}}\right) \overline{p_{i j 1}^{\mathrm{f}} p_{i j 2}^{\mathrm{f}}}+\left(1-h_{\mathrm{f}}\right)\left(1-2 p^{\mathrm{f}}\right)\left(p^{\mathrm{f}}-\overline{p_{i j 1}^{\mathrm{f}} p_{i j 2}^{\mathrm{f}}}\right)\right]}{\left(\frac{\mathrm{d} z_{i j}^{\mathrm{m}}}{\mathrm{d} \delta}-\frac{\mathrm{d} \bar{z}^{\mathrm{m}}}{\mathrm{d} \delta}\right) p_{i j}^{\mathrm{m}}}=-c_{\mathrm{m}}\left[\left(1-p^{\mathrm{m}}\right) \overline{p_{i j 1}^{\mathrm{m}} p_{i j 2}^{\mathrm{m}}}+h_{\mathrm{m}}\left(1-2 p^{\mathrm{m}}\right)\left(p^{\mathrm{m}}-\overline{p_{i j 1}^{\mathrm{m}} p_{i j 2}^{\mathrm{m}}}\right)\right] .
$$

Next, we use the fact that the different averages in eqs. (A-13), (A-15), and (A-19) correspond to different probabilities of genetic identity (Roze and Rousset, 2003; Rousset, 2004; Van Cleve et al., 2010). 
For example, $\overline{p_{i j 1}^{u} p_{i j 2}^{u}}$ corresponds to the probability that two homologous gene copies randomly sampled in a juvenile of sex $u$ before dispersal are both $A$. Further, these probabilities only need to be computed under neutrality to determine allele frequency change to the first order of $\delta$ (as they are already multiplied by $\delta$ in eqs. A-11). In such circumstances, the probabilities of identity can be connected with probabilities of identity-by-descent of neutral genes, which are independent of the frequency of $A$ in the infinite island model (see Roze and Rousset, 2003 for further considerations). Take $\overline{p_{i j 1}^{u} p_{i j 2}^{u}}$ again for example. Consider the two lineages of these genes backward in time: either the two lineages have stayed in the same group and coalesced (with a probability that we denote $F_{\text {IT }}$ and which we will show later is equal in males and females, see eq. A-41) or have dispersed to different groups patches and have not coalesced (with probability $1-F_{\mathrm{IT}}$ ). The probability that these two genes are $A$ is then equal to $F_{\text {IT }} p^{u}+\left(1-F_{\text {IT }}\right)\left(p^{u}\right)^{2}$, where $p^{u}$ is the frequency of $A$ in the population of individuals of sex $u$. However, this frequency is equal in males and females under neutrality $\left(p^{\mathrm{m}}=p^{\mathrm{f}}=p\right)$ so that we have

$$
\overline{p_{i j 1}^{u} p_{i j 2}^{u}}=F_{\mathrm{IT}} p+\left(1-F_{\mathrm{IT}}\right) p^{2}+\mathscr{O}(\delta) .
$$

The probability $F_{\mathrm{IT}}$ is thus equivalent to Wright's coefficient of inbreeding in the infinite island model. We will later compute this coefficient explicitly in terms of demographic parameters in section A.2.7. First, we express the other two averages featuring in eqs. (A-13) and (A-15) $\left(\overline{p_{i 0}^{u} p_{i j}^{u}}\right.$ and $\overline{\left.p_{i 01}^{u} p_{i 02}^{u} p_{i j}^{u}\right)}$ in terms of coalescence probabilities.

The probability, $\overline{p_{i 0}^{u} p_{i j}^{u}}$, that two genes randomly sampled in different juveniles of the same sex $u$ within the same group are both $A$ can be expressed as

$$
\overline{p_{i 0}^{u} p_{i j}^{u}}=F_{\mathrm{ST}} p+\left(1-F_{\mathrm{ST}}\right) p^{2}+\mathscr{O}(\delta),
$$

where $F_{\mathrm{ST}}$ is the probability that the two lineages of our two genes have stayed in the same group and coalesced (which is equal when those two genes are sampled in males and in females, see eq. A-36 for more details). This probability is equal to Wright's $F_{\mathrm{ST}}$ within sexes in the infinite island model.

Finally, we need $\overline{p_{i 01}^{u} p_{i 02}^{u} p_{i j}^{u}}$, which corresponds to the probability that the two homologous genes of a juvenile of sex $u$ and a third gene sampled in another juvenile of the same sex from the same group are all $A$. To compute this quantity, we let $K$ be the probability that the three lineages of these genes have stayed in the same group and coalesced; and $L$ be the probability that exactly two of those have coalesced (both probabilities are insensitive as to whether genes are sampled in males or females, see eq. A-43). With this notation, we have

$$
\overline{p_{i 01}^{u} p_{i 02}^{u} p_{i j}^{u}}=K p+L p^{2}+(1-K-L) p^{3}+\mathscr{O}(\delta) .
$$

Further, from the relationships between the different coalescence events, we can write the probability $L$ that exactly two of the genes of interest have coalesced as $L=F_{\mathrm{IT}}-K+2\left(F_{\mathrm{ST}}-K\right)$, where $F_{\mathrm{IT}}-K$ is the probability that the two homologous gene copies coalesce together but not with the gene sampled in another juvenile, and $2\left(F_{\mathrm{ST}}-K\right)$ is the probability that the gene sampled in another juvenile and one gene from the homologous copies coalesce but not with the other copy. Substituting for $L$ into eq. (A-22), we obtain

$$
\overline{p_{i 01}^{u} p_{i 02}^{u} p_{i j}^{u}}=K p+\left(F_{\mathrm{IT}}+2 F_{\mathrm{ST}}-3 K\right) p^{2}+\left(1-F_{\mathrm{IT}}-2 F_{\mathrm{ST}}+2 K\right) p^{3}+\mathscr{O}(\delta)
$$

for the probability that the two homologous genes of a juvenile of sex $u$ and a third gene sampled in another juvenile of the same sex from the same group are all $A$.

Plugging eqs. (A-20), (A-21) and (A-23) into eqs. (A-13), (A-15) and (A-19), which are in turn substituted into eq. (A-11), we get that the change in allele frequency is given by

$$
\Delta p(p)=\frac{\delta}{2} p(1-p)\left(c_{\mathrm{f}} s_{\mathrm{f}}(p)-c_{\mathrm{m}} s_{\mathrm{m}}(p)\right)+\mathscr{O}\left(\delta^{2}\right),
$$


Flintham et al., "Dispersal and sexual antagonism"

where

$$
\begin{aligned}
s_{\mathrm{f}}(p) & =\frac{1}{2} \frac{\partial w_{i j}^{\mathrm{f}}}{\partial z_{i j}^{\mathrm{f}}}\left[\left(1-F_{\mathrm{IT}}\right) p+F_{\mathrm{IT}}+\left(1-h_{\mathrm{f}}\right)(1-2 p)\left(1-F_{\mathrm{IT}}\right)\right]+\frac{1}{2} \frac{\partial w_{i j}^{\mathrm{f}}}{\partial \bar{z}_{i 0}^{\mathrm{f}}}\left[K+2\left(F_{\mathrm{ST}}-K\right)\left(p+\left(1-h_{\mathrm{f}}\right)(1-2 p)\right)\right] \\
s_{\mathrm{m}}(p) & =\frac{1}{2} \frac{\partial w_{i j}^{\mathrm{m}}}{\partial z_{i j}^{\mathrm{m}}}\left[\left(1-F_{\mathrm{IT}}\right) p+F_{\mathrm{IT}}+h_{\mathrm{m}}(1-2 p)\left(1-F_{\mathrm{IT}}\right)\right]+\frac{1}{2} \frac{\partial w_{i j}^{\mathrm{m}}}{\partial \bar{z}_{i 0}^{\mathrm{m}}}\left[K+2\left(F_{\mathrm{ST}}-K\right)\left(p+h_{\mathrm{m}}(1-2 p)\right)\right],
\end{aligned}
$$

capture selection on the $A$ allele due to its effect in females and males, respectively. We proceed to specify these selection effects in terms of demographic parameters and sex-specific dispersal, by specifying first the relevant fitness effects (i.e. fitness derivatives in section A.2.5), and second, relevant coalescence probabilities (in section A.2.7).

\section{A.2.5 Fitness}

According to the life-cycle described in section A.1, the expected number of female and male juveniles produced by a focal female juvenile can be decomposed as

$$
w_{i j}^{\mathrm{f} \rightarrow \mathrm{f}}=w_{i j}^{\mathrm{f} \rightarrow \mathrm{m}}=s_{i j}^{\mathrm{f}} k / 2
$$

where $s_{i j}^{\mathrm{f}}$ is the probability that this female juvenile survives competition to adulthood and $k / 2$ is the number of male and female juveniles produced by an adult female. When the number of groups $n_{\mathrm{g}}$ is large and selection strength $\delta$ is small, the survival probability of a focal female is related to its phenotype $z_{i j}^{\mathrm{f}}$ (i.e. its competitiveness) according to

$$
s_{i j}^{\mathrm{f}}=\frac{\left(1-m_{\mathrm{f}}\right) n_{\mathrm{f}} z_{i j}^{\mathrm{f}}}{z_{i j}^{\mathrm{f}}+\left(n_{\mathrm{f}} k / 2-1\right)\left[\left(1-m_{\mathrm{f}}\right) \bar{z}_{i 0}^{\mathrm{f}}+m_{\mathrm{f}} \bar{z}^{\mathrm{f}}\right]}+\frac{m_{\mathrm{f}} n_{\mathrm{f}} z_{i j}^{\mathrm{f}}}{z_{i j}^{\mathrm{f}}+\left(n_{\mathrm{f}} k / 2-1\right) \bar{z}^{\mathrm{f}}},
$$

where $m_{\mathrm{f}}$ is the probability that a female juvenile disperses; $\bar{z}_{i 0}^{\mathrm{f}}$ is the average phenotype among the other female juveniles born in the focal group (i.e. excluding the focal female); and $\bar{z}^{\mathrm{f}}$ is the average female phenotype in the population. The first summand of eq. (A-27) is the probability that the focal female remains in its natal group (with probability $1-m_{\mathrm{f}}$ ) and survives, and the second, that she disperses (with probability $m_{\mathrm{f}}$ ) and survives. In both cases, her survival depends on the ratio of her competitiveness $\left(z_{i j}^{\mathrm{f}}\right)$ and the expected average competitiveness in the group she is in.

Similarly, the expected number of male and female juveniles produced by a focal male juvenile is

$$
w_{i j}^{\mathrm{m} \rightarrow \mathrm{m}}=w_{i j}^{\mathrm{m} \rightarrow \mathrm{f}}=s_{i j}^{\mathrm{m}}\left(n_{\mathrm{f}} k / 2\right) / n_{\mathrm{m}},
$$

where $s_{i j}^{\mathrm{m}}$ is the probability that this male juvenile survives to adulthood and $\left(n_{\mathrm{f}} k / 2\right) / n_{\mathrm{m}}$ is the number of male and female juveniles produced by an adult male under random mating. Mirroring eq. (A-27), the survival probability of a focal male with phenotype $z_{i j}^{\mathrm{m}}$ can be written as

$$
s_{i j}^{\mathrm{m}}=\frac{\left(1-m_{\mathrm{m}}\right) n_{\mathrm{m}} z_{i j}^{\mathrm{m}}}{z_{i j}^{\mathrm{m}}+\left(n_{\mathrm{f}} k / 2-1\right)\left[\left(1-m_{\mathrm{m}}\right) \bar{z}_{i 0}^{\mathrm{m}}+m_{\mathrm{m}} \bar{z}^{\mathrm{m}}\right]}+\frac{m_{\mathrm{m}} n_{\mathrm{m}} z_{i j}^{\mathrm{m}}}{z_{i j}^{\mathrm{m}}+\left(n_{\mathrm{f}} k / 2-1\right) \bar{z}^{\mathrm{m}}},
$$

where $m_{\mathrm{m}}$ is the probability that a male juvenile disperses; $\bar{z}_{i 0}^{\mathrm{m}}$ is the average phenotype among the other male juveniles born in the focal group; and $\bar{z}^{\mathrm{m}}$ is the average male phenotype in the population. 
Substituting eq. (A-27) into eq. (A-26) and eq. (A-29) into eq. (A-28), we obtain that total female, $w_{i j}^{\mathrm{f}}$, and male, $w_{i j}^{\mathrm{m}}$, fitness (the expected total number of juveniles produced by a focal female and male juvenile) are given by

$$
\begin{aligned}
& w_{i j}^{\mathrm{f}}=w_{i j}^{\mathrm{f} \rightarrow \mathrm{f}}+w_{i j}^{\mathrm{f} \rightarrow \mathrm{m}}=2\left(\frac{\left(1-m_{\mathrm{f}}\right) z_{i j}^{\mathrm{f}}}{\left(1-m_{\mathrm{f}}\right) \bar{z}_{i 0}^{\mathrm{f}}+m_{\mathrm{f}} \bar{z}^{\mathrm{f}}}+\frac{m_{\mathrm{f}} z_{i j}^{\mathrm{f}}}{\bar{z}^{\mathrm{f}}}\right)+\mathscr{O}(1 / k), \\
& w_{i j}^{\mathrm{m}}=w_{i j}^{\mathrm{m} \rightarrow \mathrm{f}}+w_{i j}^{\mathrm{m} \rightarrow \mathrm{m}}=2\left(\frac{\left(1-m_{\mathrm{m}}\right) z_{i j}^{\mathrm{m}}}{\left(1-m_{\mathrm{m}}\right) \bar{z}_{i 0}^{\mathrm{m}}+m_{\mathrm{m}} \bar{z}^{\mathrm{m}}}+\frac{m_{\mathrm{m}} z_{i j}^{\mathrm{m}}}{\bar{z}^{\mathrm{m}}}\right)+\mathscr{O}(1 / k),
\end{aligned}
$$

when fecundity $k$ is large, which we will henceforth assume. From eq. (A-30), the direct and indirect fitness effects of female and male competitiveness are respectively given by

$$
\frac{\partial w_{i j}^{u}}{\partial z_{i j}^{u}}=2, \quad \frac{\partial w_{i j}^{u}}{\partial \bar{z}_{i 0}^{u}}=-2\left(1-m_{u}\right)^{2},
$$

for $u \in\{\mathrm{m}, \mathrm{f}\}$. Plugging eq. (A-31) into eq. (A-25), we finally obtain main text equations (2).

\section{A.2.6 Soft Selection}

Eq. A-31 is based on the assumption that dispersal precedes selection, i.e. "hard selection". Where dispersal occurs after selection ("soft selection", Roze and Rousset, 2003; Débarre and Gandon, 2011), the survival of a focal juvenile depends only on its own phenotype and the phenotypes of individuals born in the same patch, specifically

$$
s_{i j}^{\mathrm{f}}=\frac{n_{\mathrm{f}} z_{i j}^{\mathrm{f}}}{z_{i j}^{\mathrm{f}}+\left(n_{\mathrm{f}} k / 2-1\right) \bar{z}_{i 0}^{\mathrm{f}}},
$$

and

$$
s_{i j}^{\mathrm{m}}=\frac{n_{\mathrm{m}} z_{i j}^{\mathrm{m}}}{z_{i j}^{\mathrm{m}}+\left(n_{\mathrm{f}} k / 2-1\right) \bar{z}_{i 0}^{\mathrm{m}}}
$$

(compare with eqs. A-27 and A-29).

By plugging eq. (A-32) into eq. (A-26) and eq. (A-33) into eq. (A-28), and assuming large $k$, we find the direct and indirect fitness effects of competitiveness under soft selection are simply

$$
\frac{\partial w_{i j}^{u}}{\partial z_{i j}^{u}}=2, \quad \frac{\partial w_{i j}^{u}}{\partial \bar{z}_{i 0}^{u}}=-2
$$

for $u \in\{\mathrm{m}, \mathrm{f}\}$. Substituting eq. (A-34) into eq. (A-25), we get an alternative version of main text equations (2),

$$
\begin{aligned}
s_{\mathrm{f}}(p) & =p+F_{\mathrm{IT}}(1-p)+\left(1-h_{\mathrm{f}}\right)(1-2 p)\left(1-F_{\mathrm{IT}}\right) \\
s_{\mathrm{m}}(p) & =\underbrace{p+F_{\mathrm{IT}}(1-p)}_{\text {direct effect in } A A}+\underbrace{h_{\mathrm{m}}(1-2 p)\left(1-F_{\mathrm{IT}}\right)}_{\text {direct effect in } A a}-\underbrace{\left[K+2\left(F_{\mathrm{ST}}-K\right)\left(p+\left(1-h_{\mathrm{f}}\right)(1-2 p)\right)\right]}_{\text {kin competition }}
\end{aligned} .
$$

From eqs. (A-35) we can see that under soft selection, limited dispersal will narrow the scope for sexually antagonistic polymorphism by restricting the conditions where balancing selection is favoured (as under hard selection, main text eq. 5). However, unlike the hard selection case, the strength of kin 
competition is not sex-specific here (i.e. the kin competition term is not discounted by the probability of philopatry in each sex, as in main text eqs. 2). This is because selection occurs prior to dispersal so that all individuals experience kin competition in proportion to the average level of relatedness in their natal patch, which is equal between the sexes due to random mating within groups. More broadly, this means that sex-biased dispersal does not change the nature of polymorphism under soft selection (in contrast to hard selection where competitiveness is more strongly favoured in the dispersing sex, see Fig. 3A bottom).

\section{A.2.7 Coalescence probabilities at a neutral autosomal locus}

In this section, we specify coalescence probabilities relevant for selection eq. (A-25) in terms of demographic parameters (numbers of males and females, $n_{\mathrm{m}}, n_{\mathrm{f}}$, and sex-specific dispersal, $m_{\mathrm{m}}, m_{\mathrm{f}}$ ). We do so using standard identity-by-descent arguments (e.g., Wang, 1997; Roze and Rousset, 2003; Rousset, 2004; Ramachandran et al., 2008; Van Cleve et al., 2010).

Pairwise coalescence probabilities. Consider first, $F_{\mathrm{ST}}(t+1)$, the probability that two neutral genes randomly sampled at some arbitrary generation $t+1$ in different juveniles of the same sex $u$ within the same group (before dispersal) coalesce. With probability $1 / 4$ both sampled genes are maternally inherited; with probability $1 / 4$, they are paternally inherited; and with probability $1 / 2$, one is maternally inherited and the other paternally inherited. We can then write the probability that these two genes coalesce as

$$
F_{\mathrm{ST}}(t+1)=\frac{1}{4} \theta_{\mathrm{f}}(t)+\frac{1}{4} \theta_{\mathrm{m}}(t)+\frac{1}{2} \theta_{\mathrm{f}, \mathrm{m}}(t)
$$

where $\theta_{\mathrm{f}}(t)$ and $\theta_{\mathrm{m}}(t)$ are respectively the probabilities that two maternally and paternally inherited genes coalesce; and $\theta_{\mathrm{f}, \mathrm{m}}(t)$ the probability that one paternal gene and one maternal gene (in different individuals) coalesce. Consider the first of these, $\theta_{\mathrm{f}}(t)$, the probability that two maternally inherited genes coalesce. This probability will depend on whether or not the two juveniles that carry the sampled maternal genes have the same mother: 1) If the focal juveniles have the same mother (which occurs with probability $1 / n_{\mathrm{f}}$ ), then with probability $1 / 2$ the two genes are the same copy and they coalesce with probability 1 , or, with probability $1 / 2$, the two genes are the homologous copies in the mother, in which case they coalesce with probability $F_{\mathrm{IT}}(t)$ (the probability that two homologous gene copies in a juvenile sampled at generation $t$ coalesce); 2) If the focal juveniles have different mothers (which occurs with probability $1-1 / n_{\mathrm{f}}$ ), then in order for the genes to coalesce both mothers must have remained philopatric (with probability $\left(1-m_{\mathrm{f}}\right)^{2}$ ) and their genes coalesce with probability $F_{\mathrm{ST}}(t)$. From these considerations, we thus have

$$
\theta_{\mathrm{f}}(t)=\frac{1}{n_{\mathrm{f}}}\left(\frac{1}{2}+\frac{1}{2} F_{\mathrm{IT}}(t)\right)+\frac{n_{\mathrm{f}}-1}{n_{\mathrm{f}}}\left(1-m_{\mathrm{f}}\right)^{2} F_{\mathrm{ST}}(t) .
$$

Similarly, the probability that two paternally inherited genes coalesce is

$$
\theta_{\mathrm{m}}(t)=\frac{1}{n_{\mathrm{m}}}\left(\frac{1}{2}+\frac{1}{2} F_{\mathrm{IT}}(t)\right)+\frac{n_{\mathrm{m}}-1}{n_{\mathrm{m}}}\left(1-m_{\mathrm{m}}\right)^{2} F_{\mathrm{ST}}(t),
$$

where $1 / n_{\mathrm{m}}$ is the probability that two juveniles have the same father. Finally, for one paternal gene and one maternal gene to coalesce, both the mother and father of the relevant juveniles must have remained philopatric, so that

$$
\theta_{\mathrm{f}, \mathrm{m}}(t)=\left(1-m_{\mathrm{f}}\right)\left(1-m_{\mathrm{m}}\right) F_{\mathrm{ST}}(t) .
$$


Flintham et al., "Dispersal and sexual antagonism"

Substituting eqs. (A-37)-(A-39) into eq. (A-36), we obtain a recurrence for $F_{\mathrm{ST}}(t)$,

$$
\begin{aligned}
F_{\mathrm{ST}}(t+1)= & \frac{1}{4}\left(\frac{1}{n_{\mathrm{f}}}\left(\frac{1}{2}+\frac{1}{2} F_{\mathrm{IT}}(t)\right)+\frac{n_{\mathrm{f}}-1}{n_{\mathrm{f}}}\left(1-m_{\mathrm{f}}\right)^{2} F_{\mathrm{ST}}(t)\right) \\
& +\frac{1}{4}\left(\frac{1}{n_{\mathrm{m}}}\left(\frac{1}{2}+\frac{1}{2} F_{\mathrm{IT}}(t)\right)+\frac{n_{\mathrm{m}}-1}{n_{\mathrm{m}}}\left(1-m_{\mathrm{m}}\right)^{2} F_{\mathrm{ST}}(t)\right)+\frac{1}{2}\left(1-m_{\mathrm{f}}\right)\left(1-m_{\mathrm{m}}\right) F_{\mathrm{ST}}(t)
\end{aligned}
$$

that also depends on $F_{\mathrm{IT}}(t)$, the probability that two homologous gene copies in a juvenile sampled at generation $t$ coalesce. This probability, meanwhile, satisfies the recurrence

$$
F_{\mathrm{IT}}(t+1)=\left(1-m_{\mathrm{f}}\right)\left(1-m_{\mathrm{m}}\right) F_{\mathrm{ST}}(t),
$$

as for the two homologous gene copies of a juvenile to coalesce, both its mother and father must have remained philopatric, in which case the coalesce with probability $F_{\mathrm{ST}}(t)$.

Solving for the equilibrium, $F_{\mathrm{ST}}(t+1)=F_{\mathrm{ST}}(t)=F_{\mathrm{ST}}$ and $F_{\mathrm{IT}}(t+1)=F_{\mathrm{IT}}(t+1)=F_{\mathrm{IT}}$, using eqs. (A-40)(A-41), we obtain

$$
\begin{aligned}
F_{\mathrm{ST}} & =\frac{n_{\mathrm{f}}+n_{\mathrm{m}}}{n_{\mathrm{f}}\left(1-m_{\mathrm{m}}\right)\left(1+m_{\mathrm{f}}-2 m_{\mathrm{m}}\right)+n_{\mathrm{m}}\left(1-m_{\mathrm{f}}\right)\left(1+m_{\mathrm{m}}-2 m_{\mathrm{f}}\right)+2 n_{\mathrm{f}} n_{\mathrm{m}}\left(4-m_{\mathrm{f}}-m_{\mathrm{m}}\right)\left(m_{\mathrm{f}}+m_{\mathrm{m}}\right)} \\
F_{\mathrm{IT}} & =\left(1-m_{\mathrm{f}}\right)\left(1-m_{\mathrm{m}}\right) F_{\mathrm{ST}} .
\end{aligned}
$$

Substituting for $m_{\mathrm{f}}=M_{\mathrm{f}} / n_{\mathrm{f}}, m_{\mathrm{m}}=M_{\mathrm{m}} / n_{\mathrm{m}}, n_{\mathrm{f}}=(1-r) n$ and $n_{\mathrm{m}}=r n$ in the above equation, we obtain $F$ in eq. (4) of the main text in the limit $n \rightarrow \infty$ (which is equivalent to e.g. eq. 8 of Ramachandran et al., 2008).

Threeway coalescence probabilities. To compute the probability, $K$, that the two homologous genes of a juvenile (which we label as juvenile " 1 " for the sake of argument) and a third gene sampled in another juvenile (labelled juvenile "2") of the same sex sampled from the same group before dispersal all coalesce, consider that for this to happen, it is necessary that both parents of juvenile 1 are philopatric, which occurs with probability $\left(1-m_{\mathrm{f}}\right)\left(1-m_{\mathrm{m}}\right)$. Then, with probability $1 / 2$, the gene sampled in juvenile 2 is maternally inherited, in which case all three genes coalesce with a probability we call $\Theta_{\mathrm{f}}(t)$; with probability $1 / 2$, the gene sampled in juvenile 2 is paternally inherited, in which case all three genes coalesce with a probability we call $\Theta_{\mathrm{m}}(t)$, so that

$$
K(t+1)=\left(1-m_{\mathrm{f}}\right)\left(1-m_{\mathrm{m}}\right)\left(\frac{1}{2} \Theta_{\mathrm{f}}(t)+\frac{1}{2} \Theta_{\mathrm{m}}(t)\right) .
$$

We proceed to specify $\Theta_{\mathrm{f}}(t)$ and $\Theta_{\mathrm{m}}(t)$.

Consider $\Theta_{\mathrm{f}}(t)$ first. If the gene sampled in juvenile 2 is maternally inherited, then whether it coalesces with the homologous genes of juvenile 1 depends on whether or not they have the same mother. If they have the same mother (which occurs with probability $1 / n_{\mathrm{f}}$ ), then with probability $1 / 2$, the maternal genes of juveniles 1 and 2 are the same and all three genes coalesce with probability $F_{\mathrm{ST}}(t)$, and with probability $1 / 2$, the maternal genes of juveniles 1 and 2 are different and all three genes coalesce with probability $K(t)$. If, however, juveniles have different mothers (which occurs with probability $1-1 / n_{\mathrm{f}}$ ), then for all three genes to coalesce, first the mother of juvenile 2 must be philopatric (which occurs with probability $\left(1-m_{\mathrm{f}}\right)$ ) and second, three genes sampled in three different juveniles must coalesce (we call $\kappa(t)$ the probability of this happening). The probability that two homologous genes of juvenile 1 and the maternally inherited gene of juvenile coalesce then reads as

$$
\Theta_{\mathrm{f}}(t)=\frac{1}{n_{\mathrm{f}}}\left(\frac{1}{2} F_{\mathrm{ST}}(t)+\frac{1}{2} K(t)\right)+\frac{n_{\mathrm{f}}-1}{n_{\mathrm{f}}}\left(1-m_{\mathrm{f}}\right) \kappa(t) .
$$


Flintham et al., "Dispersal and sexual antagonism"

Similarly, the probability that two homologous genes of juvenile 1 and the paternally inherited gene of juvenile coalesce then reads as

$$
\Theta_{\mathrm{m}}(t)=\frac{1}{n_{\mathrm{m}}}\left(\frac{1}{2} F_{\mathrm{ST}}(t)+\frac{1}{2} K(t)\right)+\frac{n_{\mathrm{m}}-1}{n_{\mathrm{m}}}\left(1-m_{\mathrm{m}}\right) \kappa(t) .
$$

In order to compute $K(t+1)$, we therefore need an expression for $\kappa(t)$, the probability that three genes sampled in three different juveniles before dispersal coalesce. We can follow a similar argument as the one used to derive eq. (A-40). Specifically, conditioning on which genes are sampled in each juvenile (maternal or paternal), and whether these juveniles have parents in common, we obtain

$$
\begin{aligned}
\kappa(t+1)= & \frac{1}{8}\left(\frac{1}{n_{\mathrm{f}}^{2}}\left(\frac{1}{4}+\frac{3}{4} F_{\mathrm{IT}}(t)\right)+3 \frac{n_{\mathrm{f}}-1}{n_{\mathrm{f}}^{2}}\left(1-m_{\mathrm{f}}\right)^{2}\left(\frac{1}{2} F_{\mathrm{ST}}(t)+\frac{1}{2} K(t)\right)+\frac{\left(n_{\mathrm{f}}-1\right)\left(n_{\mathrm{f}}-2\right)}{n_{\mathrm{f}}^{2}}\left(1-m_{\mathrm{f}}\right)^{3} \kappa(t)\right) \\
& +\frac{3}{8}\left(1-m_{\mathrm{f}}\right)\left(1-m_{\mathrm{m}}\right)\left(\Theta_{\mathrm{f}}(t)+\Theta_{\mathrm{m}}(t)\right) \\
& +\frac{1}{8}\left(\frac{1}{n_{\mathrm{m}}^{2}}\left(\frac{1}{4}+\frac{3}{4} F_{\mathrm{IT}}(t)\right)+3 \frac{n_{\mathrm{m}}-1}{n_{\mathrm{m}}^{2}}\left(1-m_{\mathrm{m}}\right)^{2}\left(\frac{1}{2} F_{\mathrm{ST}}(t)+\frac{1}{2} K(t)\right)+\frac{\left(n_{\mathrm{m}}-1\right)\left(n_{\mathrm{m}}-2\right)}{n_{\mathrm{m}}^{2}}\left(1-m_{\mathrm{m}}\right)^{3} \kappa(t)\right),
\end{aligned}
$$

where the first line is the probability that all three genes are maternal and coalesce (decomposed according to whether the three sampled juveniles have the same mother, with probability $1 / n_{\mathrm{f}}^{2}$, two out of three have the same mother, with probability $3\left(n_{\mathrm{f}}-1\right) / n_{\mathrm{f}}^{2}$, or when they have different mothers, with probability $\left.\left(n_{\mathrm{f}}-1\right)\left(n_{\mathrm{f}}-2\right) / n_{\mathrm{f}}^{2}\right)$; the second line is the probability that two genes are maternal and one is paternal or that one is maternal and the other two are paternal and that they coalesce; and the third line is the probability that all three genes are paternal and coalesce (decomposed according to whether the three sampled juveniles have the same father, with probability $1 / n_{\mathrm{m}}^{2}$, two out of three have the same father, with probability $3\left(n_{\mathrm{m}}-1\right) / n_{\mathrm{m}}^{2}$, or when they have different fathers, with probability $\left.\left(n_{\mathrm{m}}-1\right)\left(n_{\mathrm{m}}-2\right) / n_{\mathrm{m}}^{2}\right)$.

Substituting eqs. (A-44) and (A-45) into (A-43) and (A-46) (with $F_{\mathrm{ST}}(t)$ and $F_{\mathrm{IT}}(t)$ at equilibrium eq. A42) allows us to solve for the equilibrium coalescence probabilities $K=K(t+1)=K(t)$ and $\kappa=\kappa(t+$ $1)=\kappa(t)$. Doing so we obtain a complicated expression for $K$, which when dispersal is weak and local population size is large is given by eq. (4) of the main text (i.e. substituting for $m_{\mathrm{f}}=M_{\mathrm{f}} / n_{\mathrm{f}}$, $m_{\mathrm{m}}=M_{\mathrm{m}} / n_{\mathrm{m}}, n_{\mathrm{f}}=(1-r) n$ and $n_{\mathrm{m}}=r n$ and taking the limit $\left.n \rightarrow \infty\right)$.

\section{A.2.8 Allele frequency at a polymorphic equilibrium}

In this section we derive the equilibrium frequency $p^{*}$ of the male-detrimental, female-beneficial $A$ allele when polymorphism is favoured (i.e. eq. (5) of the main text holds). By plugging main text eq. (4) (which assumes large patches) into eqs. (1)-(2) and solving for $\Delta p\left(p^{*}\right)=0$ for $p^{*}$, we find

$$
p^{*}=p_{\circ}^{*}\left(1+F\left(c_{\mathrm{f}}-c_{\mathrm{m}}\right)\right)
$$

where $F$ is given in eq. (4) and

$$
p_{\circ}^{*}=\frac{\left(1-h_{\mathrm{f}}\right) c_{\mathrm{f}}-h_{\mathrm{m}} c_{\mathrm{m}}}{\left(1-2 h_{\mathrm{f}}\right) c_{\mathrm{f}}+\left(1-2 h_{\mathrm{m}}\right) c_{\mathrm{m}}}
$$

is the equilibrium frequency in a well-mixed population (see e.g. Box 3 in Mullon et al., 2012). Eq. (A-47) reveals that, compared with the well mixed case (when $F=0$ ), limited dispersal $(F>0)$ leads to a greater equilibrium frequency of the female-beneficial allele when $c_{\mathrm{f}}>c_{\mathrm{m}}$ (and vice-versa) and that this occurs irrespective of dominance effects (see also Fig. 5A for numerical solutions of $p^{*}$ in small patches). 
Flintham et al., "Dispersal and sexual antagonism"

\section{A.3 Sex-linked locus}

In this section, we derive and analyse the allele frequency change at a sex-linked locus and show that the effect of limited on allele segregation is largely the same as on autosomes (our results are summarised in section 3.1.5 of the main text). Without loss of generality, we assume that our population of interest belongs to an XY species (i.e. in which males are the heterogametic sex), so that the sexually antagonistic allele segregates on the X-chromosome. All our results can be applied to ZW species by simply exchanging male and females variables.

\section{A.3.1 Weighted allele frequency change}

Our argument follows the same argument used for an autosomal locus in section A.2. In terms of genotype and phenotype, females are specified in the same way as for an autosomal locus (eqs. A-1 and A-3). Males however are heterogametic and therefore have genotype and phenotype respectively specified by

$$
\begin{aligned}
p_{i j}^{\mathrm{m}} & =p_{i j 1}^{\mathrm{m}} \in\{0,1\} \\
z_{i j}^{\mathrm{m}} & =1-\delta c_{\mathrm{m}} p_{i j}^{\mathrm{m}} .
\end{aligned}
$$

Since males only pass their X linked genes to their daughters, the weighted allele frequency change is now given by

$$
\Delta p=\frac{1}{n_{\mathrm{g}}} \sum_{i=1}^{n_{\mathrm{g}}} \frac{1}{n_{\mathrm{f}} k / 2}\left[\alpha\left(\sum_{j=1}^{n_{\mathrm{f}} k / 2} w_{i j}^{\mathrm{f} \rightarrow \mathrm{f}} \frac{p_{i j}^{\mathrm{f}}}{2}+\sum_{j=1}^{n_{\mathrm{f}} k / 2} w_{i j}^{\mathrm{m} \rightarrow \mathrm{f}} \frac{p_{i j}^{\mathrm{m}}}{2}\right)+(1-\alpha) \sum_{j=1}^{n_{\mathrm{f}} k / 2} w_{i j}^{\mathrm{f} \rightarrow \mathrm{m}} p_{i j}^{\mathrm{f}}\right]-p
$$

(compare with eq. A-5 for autosomes). Also due to male heterogamety, females and males now have reproductive values $\alpha=2 / 3$ and $1-\alpha=1 / 3$, respectively (see eq. A-4). Substituting for these reproductive values and using the fact that $w_{i j}^{\mathrm{f} \rightarrow \mathrm{f}}=w_{i j}^{\mathrm{f} \rightarrow \mathrm{m}}=w_{i j}^{\mathrm{f}} / 2$ and $w_{i j}^{\mathrm{m} \rightarrow \mathrm{f}}=w_{i j}^{\mathrm{m}} / 2$ for our model, eq. (A-50) becomes

$$
\Delta p=\frac{1}{6 n_{\mathrm{g}}} \sum_{i=1}^{n_{\mathrm{g}}} \frac{1}{n_{\mathrm{f}} k / 2}\left[2 \sum_{j=1}^{n_{\mathrm{f}} k / 2} w_{i j}^{\mathrm{f}} p_{i j}^{\mathrm{f}}+\sum_{j=1}^{n_{\mathrm{f}} k / 2} w_{i j}^{\mathrm{m}} p_{i j}^{\mathrm{m}}\right]-p
$$

(compare with eq. A-11 for autosomes).

Substituting fitness expansions eq. (A-10) into eq. (A-52), we obtain the following expression,

$$
\begin{aligned}
& \Delta p=\frac{\delta}{6}[ 2 \frac{\partial w_{i j}^{\mathrm{f}}}{\partial z_{i j}^{\mathrm{f}}} \overline{\left(\frac{\mathrm{d} z_{i j}^{\mathrm{f}}}{d \delta}-\frac{\mathrm{d} \bar{z}^{\mathrm{f}}}{d \delta}\right) p_{i j}^{\mathrm{f}}}+2 \frac{\partial w_{i j}^{\mathrm{f}}}{\partial\left(\frac{\mathrm{d} \bar{z}_{i 0}^{\mathrm{f}}}{d \delta}-\frac{\mathrm{d} \bar{z}^{\mathrm{f}}}{d \delta}\right) p_{i j}^{\mathrm{f}}} \\
&\left.+\frac{\partial w_{i j}^{\mathrm{m}}}{\partial z_{i j}^{\mathrm{m}}} \overline{\left(\frac{\mathrm{d} z_{i j}^{\mathrm{m}}}{d \delta}-\frac{\mathrm{d} \bar{z}^{\mathrm{m}}}{d \delta}\right) p_{i j}^{\mathrm{m}}}+\frac{\partial w_{i j}^{\mathrm{m}}}{\partial \bar{z}_{i 0}^{\mathrm{m}}} \overline{\left(\frac{\mathrm{d} \bar{z}_{i 0}^{\mathrm{m}}}{d \delta}-\frac{\left.\mathrm{d} \bar{z}^{\mathrm{m}}\right)}{d \delta}\right) p_{i j}^{\mathrm{m}}}\right]+\mathscr{O}\left(\delta^{2}\right),
\end{aligned}
$$

where the upper bar means the average over all juveniles and over all groups. The female related averages are computed in the same way as we did for autosomes (see section A.2.4), giving

$$
\begin{aligned}
& \overline{\left(\frac{\mathrm{d} z_{i j}^{\mathrm{f}}}{d \delta}-\frac{\mathrm{d} \bar{z}^{\mathrm{f}}}{\mathrm{d} \delta}\right) p_{i j}^{\mathrm{f}}}=c_{\mathrm{f}} p(1-p)\left[\left(1-F_{\mathrm{IT}}\right) p+F_{\mathrm{IT}}+\left(1-h_{\mathrm{f}}\right)(1-2 p)\left(1-F_{\mathrm{IT}}\right)\right]+\mathscr{O}(\delta) \\
& \overline{\left(\frac{\mathrm{d} \bar{z}_{i 0}^{\mathrm{f}}}{\mathrm{d} \delta}-\frac{\mathrm{d} \bar{z}^{\mathrm{f}}}{\mathrm{d} \delta}\right) p_{i j}^{\mathrm{f}}}=c_{\mathrm{f}} p(1-p)\left[K^{\mathrm{f}}+2\left(F_{\mathrm{ST}}^{\mathrm{f}}-K^{\mathrm{f}}\right)\left(p+\left(1-h_{\mathrm{f}}\right)(1-2 p)\right)\right]+\mathscr{O}(\delta)
\end{aligned}
$$


where $F_{\mathrm{IT}}$ is the probability that the two homologous gene copies at a neutral X-linked locus, sampled in a random juvenile female, are IBD (note that such a probability does not exist for males as they are heterogametic); $F_{\mathrm{ST}}^{\mathrm{f}}$ denotes the probability that two neutral X-linked are IBD when these are sampled in different female juveniles of the same group; and $K^{\mathrm{f}}$ is the probability that the two homologous gene copies in one juvenile female plus one copy in another randomly sampled juvenile female from the same group are all IBD.

For male related averages, we obtain from eq. (A-49)

$$
\begin{aligned}
& \overline{\left(\frac{\mathrm{d} z_{i j}^{\mathrm{m}}}{d \delta}-\frac{\mathrm{d} \bar{z}^{\mathrm{m}}}{d \delta}\right) p_{i j}^{\mathrm{m}}}=-\delta c_{\mathrm{m}} p(1-p)+\mathscr{O}(\delta) \\
& \overline{\left(\frac{\mathrm{d} \bar{z}_{i 0}^{\mathrm{m}}}{d \delta}-\frac{\mathrm{d} \bar{z}^{\mathrm{m}}}{d \delta}\right) p_{i j}^{\mathrm{m}}}=-\delta c_{\mathrm{m}} F_{\mathrm{ST}}^{\mathrm{m}} p(1-p)+\mathscr{O}(\delta),
\end{aligned}
$$

where $F_{\mathrm{ST}}^{\mathrm{m}}$ is the probability that two neutral X-linked are IBD when these are sampled in different male juveniles of the same group defined as in the preceding paragraph (note that in contrast to the autosomal case, this probability is not necessarily equal to when genes are sampled in females, i.e. $F_{\mathrm{ST}}^{\mathrm{f}} \neq F_{\mathrm{ST}}^{\mathrm{m}}$, see section A.3.2 for more details).

Substituting eqs. (A-53) and (A-54) in eq. (A-52), we obtain that the change in weighted allele frequency at an $\mathrm{X}$-linked locus is

$$
\Delta p(p)=\frac{\delta}{3} p(1-p)\left(2 c_{\mathrm{f}} s_{\mathrm{f}}(p)-c_{\mathrm{m}} s_{\mathrm{m}}(p)\right)+\mathscr{O}\left(\delta^{2}\right),
$$

where

$$
\begin{aligned}
s_{\mathrm{f}}(p) & =\frac{1}{2} \frac{\partial w_{i j}^{\mathrm{f}}}{\partial z_{i j}^{\mathrm{f}}}\left[\left(1-F_{\mathrm{IT}}\right) p+F_{\mathrm{IT}}+\left(1-h_{\mathrm{f}}\right)(1-2 p)\left(1-F_{\mathrm{IT}}\right)\right]+\frac{1}{2} \frac{\partial w_{i j}^{\mathrm{f}}}{\partial \bar{z}_{i 0}^{\mathrm{f}}}\left[K+2\left(F_{\mathrm{ST}}^{\mathrm{f}}-K\right)\left(p+\left(1-h_{\mathrm{f}}\right)(1-2 p)\right)\right] \\
s_{\mathrm{m}}(p) & =\frac{1}{2} \frac{\partial w_{i j}^{\mathrm{m}}}{\partial z_{i j}^{\mathrm{m}}}+\frac{1}{2} \frac{\partial w_{i j}^{\mathrm{m}}}{\partial \bar{z}_{i 0}^{\mathrm{m}}} F_{\mathrm{ST}}^{\mathrm{m}} .
\end{aligned}
$$

In turn, plugging fitness derivatives eq. (A-31) into eq. (A-56), we obtain

$$
\begin{aligned}
s_{\mathrm{f}}(p) & =\left(1-F_{\mathrm{IT}}\right) p+F_{\mathrm{IT}}+\left(1-h_{\mathrm{f}}\right)(1-2 p)\left(1-F_{\mathrm{IT}}\right)-\left(1-m_{\mathrm{f}}\right)^{2}\left[K^{\mathrm{f}}+2\left(F_{\mathrm{ST}}^{\mathrm{f}}-K^{\mathrm{f}}\right)\left(p+\left(1-h_{\mathrm{f}}\right)(1-2 p)\right)\right] \\
s_{\mathrm{m}}(p) & =1-\left(1-m_{\mathrm{m}}\right)^{2} F_{\mathrm{ST}}^{\mathrm{m}} .
\end{aligned}
$$

While the general form $s_{\mathrm{f}}(p)$ is unchanged from the autosomal case (see eq. 2 of main text), $s_{\mathrm{m}}(p)$ no longer includes direct or indirect effects arising from male heterozygotes as male hemizygosity precludes the existence of male heterozygotes. Thus, the effect of selection in males on allele frequency change, $c_{\mathrm{m}} \times s_{\mathrm{m}}(p)$ in eq. (A-55), depends only on $c_{\mathrm{m}}$ and the strength of male kin competition (determined by $\left(1-m_{\mathrm{m}}\right)^{2} F_{\mathrm{ST}}^{\mathrm{m}}$ in eq. A-57). To analyse allele dynamics at an X-linked locus further, we compute the relevant coalescence probabilities in the next section.

\section{A.3.2 Coalescence probabilities at a neutral X-linked locus}

In this section, we calculate the coalescence probabilities that are relevant for selection (eq. A-56) at an X-linked locus, using similar arguments as those used for an autosomal locus (see section A.2.7). 
Pairwise coalescence probabilities. Let us first consider the probabilities that two neutral X-linked genes sampled at generation $t+1$ in two neighbouring juvenile females, $F_{\mathrm{ST}}^{\mathrm{f}}(t+1)$, and males, $F_{\mathrm{ST}}^{\mathrm{m}}(t+$ 1 ), coalesce. As for the autosomal case (see eq. A-36), we can decompose $F_{\mathrm{ST}}^{\mathrm{f}}(t+1)$ according to whether the two sampled genes in females are either both of maternal or paternal origin (in which case they coalesce with respective probabilities $\theta_{\mathrm{f}}(t)$ and $\left.\theta_{\mathrm{m}}(t)\right)$ or one is of maternal and the other of paternal origin (in which case they coalesce with respective probabilities $\theta_{\mathrm{f}, \mathrm{m}}(t)$ and $\theta_{\mathrm{m}}(t)$ ). For Xlinked genes sampled in two different males, $F_{\mathrm{ST}}^{\mathrm{m}}(t+1)$, both genes are necessarily of maternal origin. We thus obtain a recurrence of the form

$$
\begin{aligned}
& F_{\mathrm{ST}}^{\mathrm{f}}(t+1)=\frac{1}{4} \theta_{\mathrm{f}}(t)+\frac{1}{4} \theta_{\mathrm{m}}(t)+\frac{1}{2} \theta_{\mathrm{f}, \mathrm{m}}(t) \\
& F_{\mathrm{ST}}^{\mathrm{m}}(t+1)=\theta_{\mathrm{f}}(t) .
\end{aligned}
$$

Then, coalescence probabilities according to parental origin can be decomposed as

$$
\begin{aligned}
\theta_{\mathrm{f}}(t) & =\frac{1}{n_{\mathrm{f}}}\left(\frac{1}{2}+\frac{1}{2} F_{\mathrm{IT}}(t)\right)+\frac{n_{\mathrm{f}}-1}{n_{\mathrm{f}}}\left(1-m_{\mathrm{f}}\right)^{2} F_{\mathrm{ST}}^{\mathrm{f}}(t) \\
\theta_{\mathrm{m}}(t) & =\frac{1}{n_{\mathrm{m}}}+\frac{n_{\mathrm{m}}-1}{n_{\mathrm{m}}}\left(1-m_{\mathrm{m}}\right)^{2} F_{\mathrm{ST}}^{\mathrm{m}}(t) \\
\theta_{\mathrm{f}, \mathrm{m}}(t) & =\left(1-m_{\mathrm{f}}\right)\left(1-m_{\mathrm{m}}\right) F_{\mathrm{ST}}^{\mathrm{f}, \mathrm{m}}(t),
\end{aligned}
$$

where $F_{\mathrm{IT}}(t)$ is the probability that the two homologous gene copies at a neutral X-linked locus in a randomly juvenile female at generation $t$ coalesce, and $F_{\mathrm{ST}}^{\mathrm{f}, \mathrm{m}}(t)$ is the coalescence probability for two X-linked genes, one sampled in a juvenile male and another in a juvenile female (from the same group) at generation $t$. In turn, these two probabilities satisfy,

$$
\begin{aligned}
F_{\mathrm{IT}}(t+1) & =\theta_{\mathrm{f}, \mathrm{m}}(t) \\
F_{\mathrm{ST}}^{\mathrm{f}, \mathrm{m}}(t+1) & =\frac{1}{2} \theta_{\mathrm{f}}(t)+\frac{1}{2} \theta_{\mathrm{f}, \mathrm{m}}(t) .
\end{aligned}
$$

Plugging eq. (A-59) into eqs. (A-58) and (A-60) and solving simultaneously for the equilibrium $F_{\mathrm{ST}}^{\mathrm{f}}=$ $F_{\mathrm{ST}}^{\mathrm{f}}(t+1)=F_{\mathrm{ST}}^{\mathrm{f}}(t), F_{\mathrm{ST}}^{\mathrm{m}}=F_{\mathrm{ST}}^{\mathrm{m}}(t+1)=F_{\mathrm{ST}}^{\mathrm{m}}(t), F_{\mathrm{ST}}^{\mathrm{f}, \mathrm{m}}=F_{\mathrm{ST}}^{\mathrm{f}, \mathrm{m}}(t+1)=F_{\mathrm{ST}}^{\mathrm{f}, \mathrm{m}}(t)$, and $F_{\mathrm{IT}}=F_{\mathrm{IT}}(t+1)=F_{\mathrm{IT}}(t)$ allows us to specify the relevant coalescence probabilities for selection on an additive allele $\left(h_{\mathrm{f}}=1 / 2\right)$ in terms of demographic parameters and sex-specific dispersal. Because such a calculation leads to complicated (but analytical) expressions, we only present their approximation when patches are large and dispersal is weak in the main text (by substituting for $m_{\mathrm{f}}=M_{\mathrm{f}} / n_{\mathrm{f}}, m_{\mathrm{m}}=M_{\mathrm{m}} / n_{\mathrm{m}}, n_{\mathrm{f}}=(1-r) n$, and $n_{\mathrm{m}}=r n$ and taking the limit $n \rightarrow \infty$, in which case we find $F_{\mathrm{X}}=F_{\mathrm{IT}}=F_{\mathrm{ST}}^{\mathrm{m}}=F_{\mathrm{ST}}^{\mathrm{f}}$ given by eq. (7) in the main text, which is equivalent to eq. 9 of Ramachandran et al., 2008).

Threeway coalescence probabilities. We now develop expressions for threeway coalescence probabilities. Recall that for selection (eq. A-56), we seek to compute $K^{\mathrm{f}}$, which is the probability that the two homologous gene copies in one juvenile female plus one copy in another randomly sampled juvenile female from the same group are all IBD. We further introduce $K^{\mathrm{m}}$, which is the probability that the two homologous gene copies in one juvenile female plus one copy in a randomly sampled juvenile male from the same group are IBD, and which will be necessary in our calculations. Using a similar argument as for the autosomal case (see eq. A-43), we can write,

$$
\begin{aligned}
K^{\mathrm{f}}(t+1) & =\left(1-m_{\mathrm{f}}\right)\left(1-m_{\mathrm{m}}\right)\left(\frac{1}{2} \Theta_{\mathrm{f}}(t)+\frac{1}{2} \Theta_{\mathrm{m}}(t)\right) \\
K^{\mathrm{m}}(t+1) & =\left(1-m_{\mathrm{f}}\right)\left(1-m_{\mathrm{m}}\right) \Theta_{\mathrm{f}}(t),
\end{aligned}
$$


where $\Theta_{\mathrm{f}}(t)$ is the probability that all three relevant genes coalesce when the one gene sampled as a single copy is maternally inherited, and $\Theta_{\mathrm{m}}(t)$ when it is paternally inherited. These probabilities can be expressed as

$$
\begin{aligned}
\Theta_{\mathrm{f}}(t) & =\frac{1}{n_{\mathrm{f}}}\left(\frac{1}{2} F_{\mathrm{ST}}^{\mathrm{f}, \mathrm{m}}+\frac{1}{2} K^{\mathrm{m}}(t)\right)+\frac{n_{\mathrm{f}}-1}{n_{\mathrm{f}}}\left(1-m_{\mathrm{f}}\right) \kappa^{\mathrm{ffm}}(t) \\
\Theta_{\mathrm{m}}(t) & =\frac{1}{n_{\mathrm{m}}} F_{\mathrm{ST}}^{\mathrm{f}, \mathrm{m}}+\frac{n_{\mathrm{m}}-1}{n_{\mathrm{m}}}\left(1-m_{\mathrm{m}}\right) \kappa^{\mathrm{mmf}}(t),
\end{aligned}
$$

where $\kappa^{\mathrm{ffm}}(t)$ is the probability that three genes, randomly sampled in two different female and one male juvenile from the same group all coalesce, and $\kappa^{\mathrm{mmf}}(t)$ is the coalescence probability for genes randomly sampled in two different male and one female juvenile from the same group. In turn, such probabilities can be written as

$$
\begin{aligned}
\kappa^{\mathrm{ffm}}(t+1)= & \frac{1}{4}\left(\frac{1}{n_{\mathrm{f}}^{2}}\left(\frac{1}{4}+\frac{3}{4} F_{\mathrm{IT}}(t)\right)+3 \frac{n_{\mathrm{f}}-1}{n_{\mathrm{f}}^{2}}\left(1-m_{\mathrm{f}}\right)^{2}\left(\frac{1}{2} F_{\mathrm{ST}}^{\mathrm{f}}(t)+\frac{1}{2} K^{\mathrm{f}}(t)\right)+\frac{\left(n_{\mathrm{f}}-1\right)\left(n_{\mathrm{f}}-2\right)}{n_{\mathrm{f}}^{2}}\left(1-m_{\mathrm{f}}\right)^{3} \kappa^{\mathrm{fff}}(t)\right) \\
& +\frac{1}{4}\left(1-m_{\mathrm{f}}\right)\left(1-m_{\mathrm{m}}\right)\left(2 \Theta_{\mathrm{f}}(t)+\Theta_{\mathrm{m}}(t)\right) \\
\kappa^{\mathrm{mmf}}(t+1)= & \frac{1}{2}\left(\frac{1}{n_{\mathrm{f}}^{2}}\left(\frac{1}{4}+\frac{3}{4} F_{\mathrm{IT}}(t)\right)+3 \frac{n_{\mathrm{f}}-1}{n_{\mathrm{f}}^{2}}\left(1-m_{\mathrm{f}}\right)^{2}\left(\frac{1}{2} F_{\mathrm{ST}}^{\mathrm{f}}(t)+\frac{1}{2} K^{\mathrm{f}}(t)\right)+\frac{\left(n_{\mathrm{f}}-1\right)\left(n_{\mathrm{f}}-2\right)}{n_{\mathrm{f}}^{2}}\left(1-m_{\mathrm{f}}\right)^{3} \kappa^{\mathrm{fff}}(t)\right) \\
& +\frac{1}{2}\left(1-m_{\mathrm{f}}\right)\left(1-m_{\mathrm{m}}\right) \Theta_{\mathrm{f}}(t),
\end{aligned}
$$

which depend on $\kappa^{\mathrm{fff}}(t)$ and $\kappa^{\mathrm{mmm}}(t)$, the coalescence probabilities for genes randomly sampled in three different female and male juveniles, respectively. In turn, these two latter probabilities themselves satisfy the recurrences

$$
\begin{aligned}
\kappa^{\mathrm{fff}}(t+1)= & \frac{1}{8}\left(\frac{1}{n_{\mathrm{f}}^{2}}\left(\frac{1}{4}+\frac{3}{4} F_{\mathrm{IT}}(t)\right)+3 \frac{n_{\mathrm{f}}-1}{n_{\mathrm{f}}^{2}}\left(1-m_{\mathrm{f}}\right)^{2}\left(\frac{1}{2} F_{\mathrm{ST}}^{\mathrm{f}}(t)+\frac{1}{2} K^{\mathrm{f}}(t)\right)+\frac{\left(n_{\mathrm{f}}-1\right)\left(n_{\mathrm{f}}-2\right)}{n_{\mathrm{f}}^{2}}\left(1-m_{\mathrm{f}}\right)^{3} \kappa^{\mathrm{fff}}(t)\right) \\
& +\frac{3}{8}\left(1-m_{\mathrm{f}}\right)\left(1-m_{\mathrm{m}}\right)\left(\Theta_{\mathrm{f}}(t)+\Theta_{\mathrm{m}}(t)\right) \\
& +\frac{1}{8}\left(\frac{1}{n_{\mathrm{m}}^{2}}+3 \frac{n_{\mathrm{m}}-1}{n_{\mathrm{m}}^{2}}\left(1-m_{\mathrm{m}}\right)^{2} F_{\mathrm{ST}}^{\mathrm{f}, \mathrm{m}}(t)+\frac{\left(n_{\mathrm{m}}-1\right)\left(n_{\mathrm{m}}-2\right)}{n_{\mathrm{m}}^{2}}\left(1-m_{\mathrm{m}}\right)^{3} \kappa^{\mathrm{mmm}}(t)\right) \\
\kappa^{\mathrm{mmm}}(t+1)= & \frac{1}{n_{\mathrm{f}}^{2}}\left(\frac{1}{4}+\frac{3}{4} F_{\mathrm{IT}}(t)\right)+3 \frac{n_{\mathrm{f}}-1}{n_{\mathrm{f}}^{2}}\left(1-m_{\mathrm{f}}\right)^{2}\left(\frac{1}{2} F_{\mathrm{ST}}^{\mathrm{f}}(t)+\frac{1}{2} K^{\mathrm{f}}(t)\right)+\frac{\left(n_{\mathrm{f}}-1\right)\left(n_{\mathrm{f}}-2\right)}{n_{\mathrm{f}}^{2}}\left(1-m_{\mathrm{f}}\right)^{3} \kappa^{\mathrm{fff}}(t) .
\end{aligned}
$$

Substituting eqs. (A-62) into eqs. (A-61),(A-63) and (A-64) finally yields a dynamical system whose equilibrium we can solve to obtain the relevant quantity, $K^{\mathrm{f}}=K^{\mathrm{f}}(t+1)=K^{\mathrm{f}}(t)$, for selection. In the high density-low migration limit (letting $m_{\mathrm{f}}=M_{\mathrm{f}} / n_{\mathrm{f}}, m_{\mathrm{m}}=M_{\mathrm{m}} / n_{\mathrm{m}}, n_{\mathrm{f}}=(1-r) n$ and $n_{\mathrm{m}}=r n$ and taking the limit $n \rightarrow \infty$ ), we obtain

$$
K^{\mathrm{f}}=\frac{F_{\mathrm{X}}(1+r)}{1+r+3\left(M_{\mathrm{m}}(1-r)+2 M_{\mathrm{f}} r\right)}
$$

where $F_{\mathrm{X}}$ is given by eq. (7) in the main text.

\section{A.3.3 Analysis of allele frequency change at a sex-linked locus}

In this section, we analyse eqs. (A-55)-(A-57) using eqs. (7) and (A-65) assuming first additive and then non-additive allele effects, in order to understand the effects of limited dispersal on the segregation 
of sexually antagonistic alleles on the X-chromosome.

Additive effects. To obtain eq. (6) of the main text, we substitute eq. (7) into eqs. (A-55)-(A-57) with $h_{\mathrm{f}}=1 / 2$ and $c_{\mathrm{f}}=c_{\mathrm{m}}=c$.

Non-additive effects. Plugging eqs. (7) and (A-65) into eqs. (A-55)-(A-57) and deriving conditions such that $\Delta p^{\prime}(0)>0$ and $\Delta p^{\prime}(1)<0$, we find that the conditions favouring the maintenance of polymorphism under non-additive effects are

$$
\frac{1}{2\left(1-h_{\mathrm{f}}\right)}\left(1+\frac{(1+r)\left(1-2 h_{\mathrm{f}}\right)}{1+r+6\left(1-h_{\mathrm{f}}\right)\left(M_{\mathrm{m}}(1-r)+2 M_{\mathrm{f}} r\right)}\right)<\frac{c_{\mathrm{f}}}{c_{\mathrm{m}}}<\frac{1}{2 h_{\mathrm{f}}}\left(1-\frac{(1+r)\left(1-2 h_{\mathrm{f}}\right)}{1+r+6 h_{\mathrm{f}}\left(M_{\mathrm{m}}(1-r)+2 M_{\mathrm{f}} r\right)}\right) .
$$

Note that if males and females are not dispersal limited (i.e. when $M_{\mathrm{m}}$ and $M_{\mathrm{f}}$ tend to infinity), eq. (A-66) reduces to the polymorphism condition of a well-mixed population (e.g. eq. 2 in Fry, 2010, under weak selection). However, as increasingly limited dispersal leads to inbreeding and a dearth of female heterozygotes, the polymorphism space contracts (see left and right sides of the inequality A66 as $M_{\mathrm{m}}$ and $M_{\mathrm{f}}$ decrease). Furthermore, because coalescence occurs faster on the X-chromosome than autosomes (main text eqs. 4 and 7), this narrowing of polymorphism conditions occurs more rapidly for X-linked sexually antagonistic loci (compare Fig. 3A and B Top).

As with autosomes, sex-biased dispersal at X-linked loci leads one sex to experience stronger kin competition and shifts the polymorphism space in favour of stronger antagonistic effects in the less dispersive sex (Fig. 3B Bottom). Notice, however, that the size of polymorphism conditions on the Xchromosome are affected by the immigrant sex ratio even when there are equal numbers of males and females $(r=0.5)$, becoming smaller when immigrants are male-biased. This is because coalescence probabilities (and thus frequency of inbreeding) on the X-chromosome are increased when males are the more dispersive sex (eq. 7).

Finally, solving for $\Delta p\left(p^{*}\right)=0$ (from eqs. A-55-A-57 with eqs. 7 and A-65), we find that when eq. (A-66) holds and selection favours polymorphism, the equilibrium allele frequency $p^{*}$ on an $\mathrm{X}$-chromosome is given by

$$
p^{*}=p_{\circ X}^{*}+\frac{1+r}{3\left[M_{\mathrm{m}}(1-r)+2 M_{\mathrm{f}} r\right]} \times \frac{c_{\mathrm{f}}-c_{\mathrm{m}}}{2\left(1-2 h_{\mathrm{f}}\right) c_{\mathrm{f}}},
$$

where

$$
p_{\circ X}^{*}=\frac{2\left(1-h_{\mathrm{f}}\right) c_{\mathrm{f}}-c_{\mathrm{m}}}{2\left(1-2 h_{\mathrm{f}}\right) c_{\mathrm{f}}}
$$

is the equilibrium in a well mixed population. Inspection of the last term of eq. (A-67) reveals that $p^{*}$ will increase or decrease depending on whether the sign of $c_{\mathrm{f}}-c_{\mathrm{m}}$ is positive or negative respectively. Thus, eq. (A-67) shows that, relative to a well-mixed population, limited dispersal (low values of $M_{\mathrm{m}}$ and $M_{\mathrm{f}}$ ) leads selection to favour the sex which feels the greatest cost of their detrimental allele independently of dominance (in this case $h_{\mathrm{f}}$ ), like on autosomes (see eq. A-47).

\section{B Individual based simulations}

We simulated a diploid population subdivided among $n_{\mathrm{g}}=50$ groups following the life-cycle elaborated in Appendix A.1 (our simulation code is available as an online enhancement to this article). 
Flintham et al., "Dispersal and sexual antagonism"

Specifically, at the beginning of a generation, adult females in each patch produce large poisson distributed number (mean $k=20$ ) of eggs. Such high female fecundity effectively precludes the possibility of patch extinction from stochastic fluctuations in the number of eggs being laid. Each egg is fertilised by a randomly sampled male within the same patch to produce a zygote whose sex is determined at random (with probability $1 / 2$ to be male and $1 / 2$ to be a female). Each zygote disperses independently with sex-specific dispersal probability $m_{\mathrm{m}}$ and $m_{\mathrm{f}}$ for males and females, respectively. If a zygote disperses, it joins a patch other than its natal one at random (so each remaining patch has a probability $1 /\left(n_{\mathrm{g}}-1\right)$ of being the destination of dispersal). After dispersal, zygotes within each patch compete intrasexually to form the new adult mating pool. To model this, $n_{\mathrm{m}}$ males and $n_{\mathrm{f}}$ females are sampled without replacement from the male and female zygotic pools in each patch, where the probability of an individual being sampled is weighted by the competitiveness of its genotype (according to Fig. 1A).

Simulations were initiated with both alleles at frequency 0.5 at Hardy-Weinberg equilibrium across the whole population and ran until one allele was fixed. 10,000 replicates were conducted for each parameter combination. For Fig. 4, we varied proportion of immigrants: $\left(M_{\mathrm{m}}+M_{\mathrm{f}}\right) /\left(n_{\mathrm{m}}+n_{\mathrm{f}}\right)=$ $10^{-2.7}, 10^{-2.6}, \ldots, 10^{-0.1}, 1$, the strength of selection: $c=c_{\mathrm{m}}=c_{\mathrm{f}}=0,0.05,0.2$ and dominance effects: $h_{\mathrm{m}}=h_{\mathrm{f}}=0.2,0.5$. Meanwhile for Fig. 5 we varied the immigrant ratio: $M_{\mathrm{m}} /\left(M_{\mathrm{m}}+M_{\mathrm{f}}\right)=$ $0.1,0.3,0.5,0.7,0.9$, and the ratio of homozygous allele costs: $c_{\mathrm{f}} / c_{\mathrm{m}}=0.25,0.82,1,1.22,4$ (for other simulation parameters, see figure legends). At the end of each generation, we calculated $F_{\mathrm{IT}}$ as

$$
F_{\mathrm{IT}}=1-\frac{\sum_{i=1}^{n_{\mathrm{g}}} \sum_{j=1}^{n} \frac{1}{n_{\mathrm{g}}} \frac{1}{n}\left[p_{i j 1}\left(1-p_{i j 2}\right)+\left(1-p_{i j 1}\right) p_{i j 2}\right]}{2 p(1-p)},
$$

where $n=n_{\mathrm{m}}+n_{\mathrm{f}}$ is the number of individuals of either sex in a given group, $p_{i j 1}$ and $p_{i j 2}$ is the frequency of $A$ in individual indexed $j$ of group $i$ at the maternally and paternally inherited locus respectively, and $p=\sum_{i=1}^{n_{\mathrm{g}}} \sum_{j=1}^{n}\left(p_{i j 1}+p_{i j 2}\right) /\left(2 n_{\mathrm{g}} n\right)$ is the frequency of allele $A$ in the whole population (so the fraction in eq. B-1 is the ratio of the observed number of heterozygotes to the expected number of heterozygotes under Hardy-Weinberg equilibrium). $F_{\mathrm{ST}}$ is calculated as the ratio of the genetic covariance between individuals within groups to half the expected total genetic variance in the population at Hardy-Weinberg equilibrium, i.e. as

$$
F_{\mathrm{ST}}=\frac{\sum_{i=1}^{n_{\mathrm{g}}} \frac{1}{n_{\mathrm{g}}}\left[\sum_{j=1}^{n} \sum_{k=j+1}^{n} \frac{2}{n(n-1)}\left(p_{i j}-p\right)\left(p_{i k}-p\right)\right]}{p(1-p)} .
$$

Eqs. B-1 and B-2 follow from eqs. A-20 and A-21 respectively, but are calculated in adults. To generate the points in Figure $4 \mathrm{~B}$, we averaged $F_{\mathrm{IT}}$ and $F_{\mathrm{ST}}$ calculations across all generations in a simulation run, with the exception of the last five (as one allele would be very close to fixation), and then across all replicate runs of a given parameter combination. 
Flintham et al., "Dispersal and sexual antagonism"
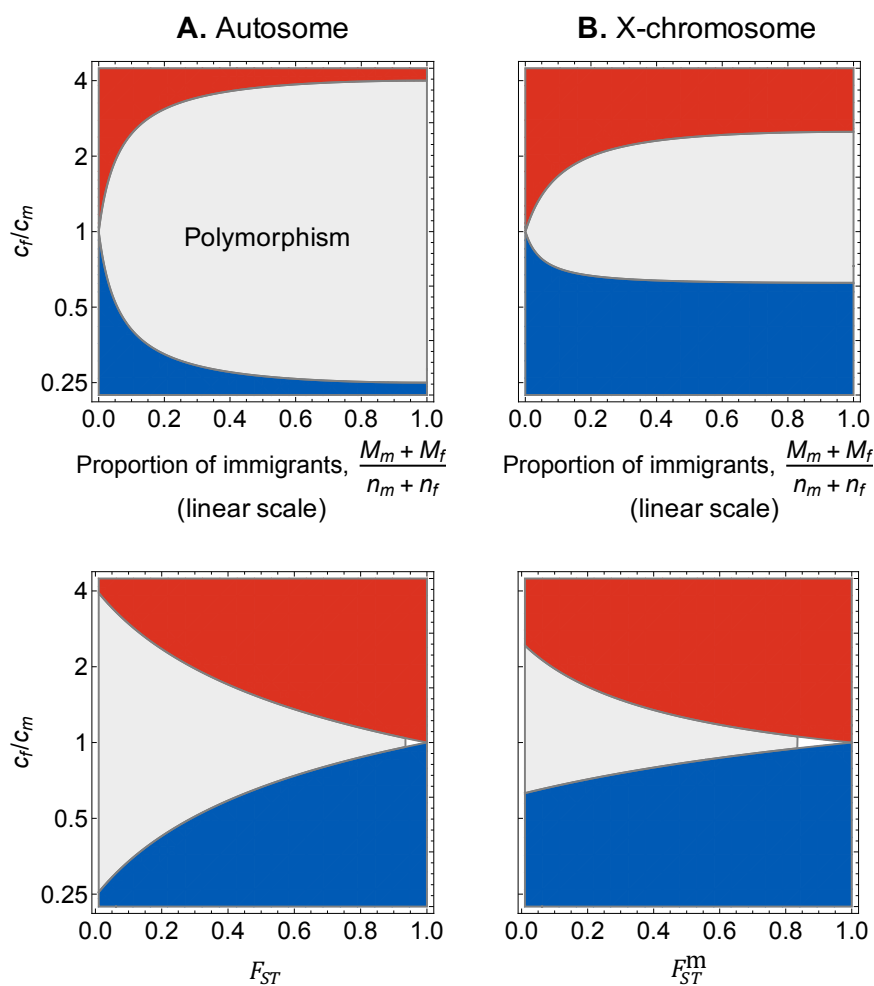

Supplementary Figure 1 : Parameters that favour the maintenance of sexually antagonistic variation and association with $F$-statistics. Combinations of parameters that lead to either balancing selection (grey, when $\Delta p^{\prime}(0)>0$ and $\Delta p^{\prime}(1)<0$ ), positive selection for $A$ (red, when $\Delta p^{\prime}(p)>0$ for all $p$ ), and positive selection for $a$ (blue, when $\Delta p^{\prime}(p)<0$ for all $p$ ) at A. an autosomal locus (computed from eqs. 1-2 with exact coalescence probabilities, see Appendix A.2.7 for calculations of these probabilities) and B. X-linked locus (computed from eqs. A-55-A-57 with exact coalescence probabilities, see Appendix A.3.2 for calculations). Top: selection according to ratio of homozygotic effects in females and males $\left(c_{\mathrm{m}} / c_{\mathrm{f}}\right)$ and the expected proportion of immigrants in each patch at each generation $\left(\left(M_{\mathrm{m}}+M_{\mathrm{f}}\right) /\left(n_{\mathrm{m}}+n_{\mathrm{f}}\right)\right.$ (on a linear scale); other parameters: $n_{\mathrm{m}}=n_{\mathrm{f}}=5, h_{\mathrm{f}}=h_{\mathrm{m}}=0.2, M_{\mathrm{m}}=M_{\mathrm{f}}$ ). Bottom: selection according to ratio of homozygotic effects in females and males $\left(c_{\mathrm{m}} / c_{\mathrm{f}}\right)$ and relevant $F_{\mathrm{ST}}$ (here shown among males for the X-chromosome) when patches are large $\left(n_{\mathrm{m}}+n_{\mathrm{f}}=100\right)$ and dispersal is symmetric among the sexes $\left(M_{\mathrm{m}}=M_{\mathrm{f}}\right.$; other parameters: $\left.h_{\mathrm{f}}=h_{\mathrm{m}}=0.2\right)$. As described in section 3.1.3, the parameter conditions which favour polymorphism become more restrictive with limited dispersal, and therefore with increasing $F_{\mathrm{ST}}$, on both autosomes and X-chromosomes. Note that this effect also occurs regardless of whether group size is small or large (top versus bottom panels), as the most relevant quantities for determining genetic structure are the effective numbers of male and female immigrants in a patch $\left(M_{\mathrm{m}}\right.$ and $M_{\mathrm{f}}$, see eqs. 4 and A-42). 

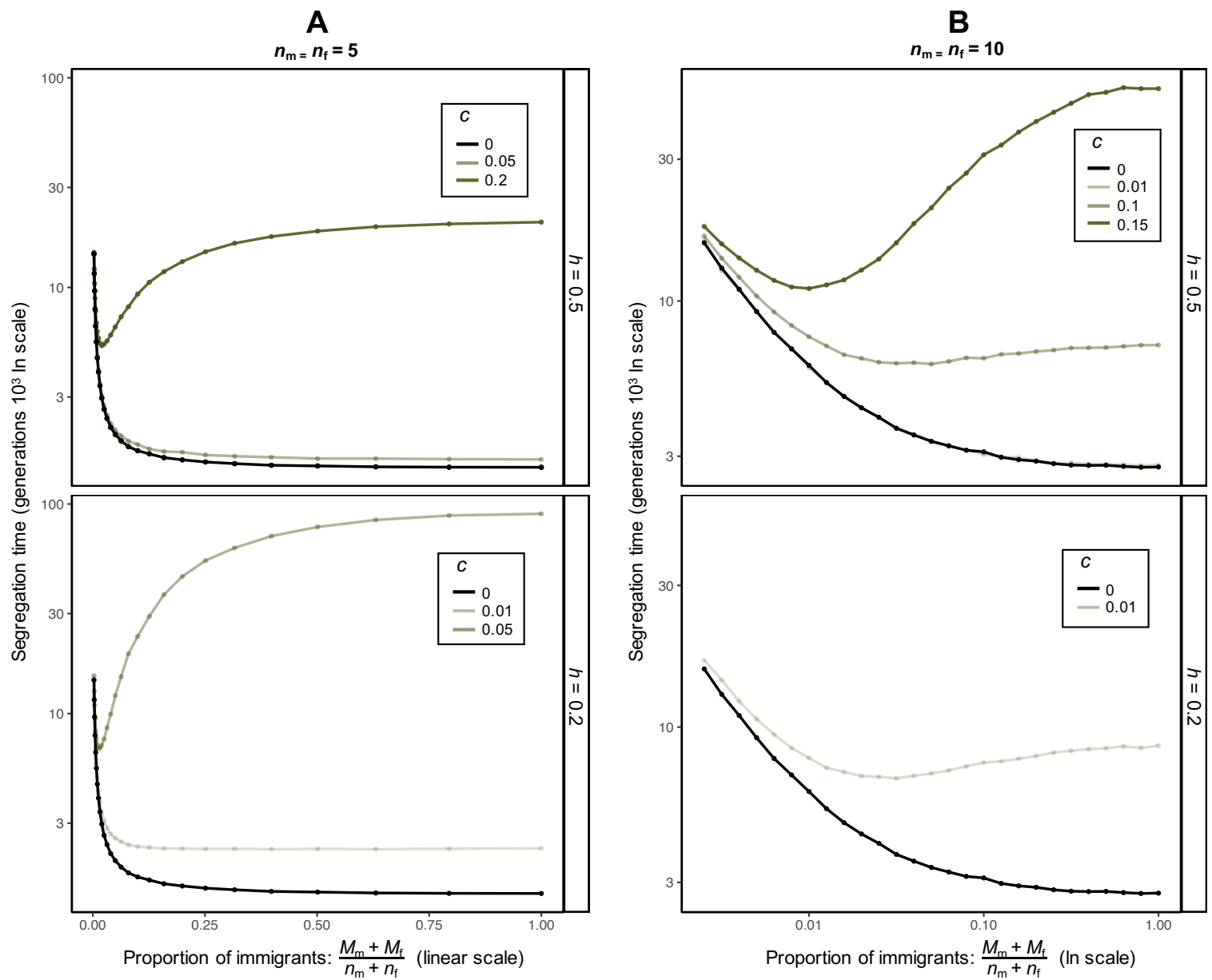

Supplementary Figure 2 : Additional plots showing allele segregation time in individualbased simulations. Segregation time averaged across replicate runs as a function of total fraction of immigrants, selection strength $\left(c=c_{\mathrm{f}}=c_{\mathrm{m}}\right)$, and dominance regime $\left(h=h_{\mathrm{f}}=h_{\mathrm{m}}\right)$. A. Shows segregation time for small groups $\left(n_{\mathrm{m}}=n_{\mathrm{f}}=5\right.$, as in Fig. 4) on a linear scale (black line shows neutrality, green lines show, from lightest to darkest, $c=0.01, c=0.05$ and $c=0.2$ ). B. Shows segregation time for larger groups $\left(n_{\mathrm{m}}=n_{\mathrm{f}}=10\right)$ on a natural $\log$ scale (black line shows neutrality, green lines show, from lightest to darkest, $c=0.01, c=0.1$ and $c=0.15$, note that black and lightest green lines overlay each under when $h=0.5$ ). See Appendix B for more details on simulation procedure. 